\title{
An extension of the SHARC survey ${ }^{\star} \star \star$
}

\author{
C. Adami ${ }^{1}$, M. P. Ulmer ${ }^{2}$, F. Durret ${ }^{3,4}$, G. Covone ${ }^{5}$, E. Cypriano ${ }^{6}$, B. P. Holden ${ }^{7}$, R. Kron ${ }^{8}$, \\ G. B. Lima Neto ${ }^{9}$, A. K. Romer ${ }^{10}$, D. Russeil ${ }^{1}$, and B. Wilhite ${ }^{11}$
}

\author{
1 LAM, Traverse du Siphon, 13012 Marseille, France \\ e-mail: christophe.adami@oamp.fr \\ 2 Department Physics \& Astronomy, Northwestern University, Evanston, IL 60208-2900, USA \\ 3 Institut d'Astrophysique de Paris, CNRS, UMR 7095, Université Pierre et Marie Curie, 98bis Bd Arago, 75014 Paris, France \\ 4 Observatoire de Paris, LERMA, 61 Av. de l'Observatoire, 75014 Paris, France \\ 5 INAF - Osservatorio Astronomico di Capodimonte, Naples, Italy \\ 6 Department of Physics and Astronomy, University College London, London WC1E 6BT, UK \\ 7 UCO/Lick Observatory, University of California, Santa Cruz, CA 95064, USA \\ 8 University of Chicago, Department of Astronomy and Astrophysics, 5640 South Ellis Avenue, Chicago, IL 60637, USA \\ 9 Instituto de Astronomia, Geofísica e C. Atmosf./USP, R. do Matão 1226, 05508-090 São Paulo/SP, Brazil \\ 10 Astronomy Centre, University of Sussex, Falmer, Brighton BN1 9QJ, UK \\ 11 Department of Astronomy, National Center for Supercomputing Applications, University of Illinois, Urbana-Champaign, \\ 1002 W. Green, Urbana, IL 61801, USA
}

Received 6 February 2007 / Accepted 30 May 2007

\section{ABSTRACT}

\begin{abstract}
Aims. We report on our search for distant clusters of galaxies based on optical and X-ray follow up observations of X-ray candidates from the SHARC survey, and based on the assumption that the absence of bright optical or radio counterparts to possibly extended $\mathrm{X}$-ray sources could mean that they are distant clusters.

Methods. We have obtained deep optical images and redshifts for several of these objects and analyzed archive XMM-Newton or Chandra data where applicable.

Results. In our list of candidate clusters, two are probably galaxy structures at redshifts of $z \sim 0.51$ and 0.28 . Seven other structures are possibly galaxy clusters between $z \sim 0.3$ and 1 . Three sources are identified with QSOs and are thus likely to be X-ray point sources, and six more also probably fall in this category. One X-ray source is spurious or variable. For 17 other sources, the data are too sparse at this time to put forward any hypothesis on their nature. We also serendipitously detected a cluster at $z=0.53$ and another galaxy concentration which is probably a structure with a redshift in the [0.15-0.6] range.

Conclusions. We discuss these results within the context of future space missions to demonstrate the necessity of a wide field of view telescope optimized for the $0.5-2 \mathrm{keV}$ range.
\end{abstract}

Key words. galaxies: clusters: general - X-rays: galaxies: clusters - surveys

\section{Introduction}

Clusters of galaxies are currently used as a complementary tool to WMAP and distant supernovae to constrain cosmological parameters as well as the equation of state of dark energy (e.g. Romer et al. 2001; Allen et al. 2004). However, without strong constraints on cluster formation and evolution, the reliability of clusters as cosmological probes will remain in doubt. In particular, their formation redshift is still not well established (e.g. Andreon et al. 2004; Holden et al. 2004). This is one of the major quests of modern astronomy. The higher the redshift of the system, the younger it is, hence scientists are continually searching for higher redshift galaxy clusters. Moreover, from a very simplified point of view (probably too simple, however), the more

* Based on observations obtained at the Canada-France-Hawaii Telescope (CFHT), Gemini Observatory, Observatoire de Haute Provence, German-Spanish Astronomical Center Calar Alto, ESO at La Silla and Paranal, Apache Point Observatory 3.5-m telescope and on data taken from the SDSS, SIMBAD and NASA/IPAC Extragalactic (NED) Databases (see acknowledgments).

$\star \star$ Appendix A is only available in electronic form at http://www. aanda.org massive the cluster, the longer it takes to virialize and therefore when virialized, the older it is (e.g. Sarazin 1986). A good way to detect clusters is to use X-rays (a not exhaustive reference list is: Burke et al. 1997; Henry et al. 1997; Scharf et al. 1997; Ebeling et al. 1998; Rosati et al. 1998; Vikhlinin et al. 1998; Nichol et al. 1999; Romer et al. 2000; Pierre et al. 2006). This is because rich clusters should have a hot intra cluster medium (ICM hereafter), which is detectable as a thermal X-ray source against the relatively faint diffuse X-ray background.

$\mathrm{X}$-ray searches for distant rich clusters have generally required that the X-ray sources be detected as extended. In all cases, however, the X-ray sources were followed up by optical imaging and spectroscopy (see e.g. the Bright-SHARC survey: Romer et al. 2000) in order to obtain redshifts and to characterize the galaxies and richness of the clusters. Unfortunately, not all clusters appear as extended X-ray sources if the point spread function of the X-ray telescope has been degraded, which can be the case when images are far off axis. Furthermore, the $\mathrm{X}$-ray emission of distant clusters and groups may have relatively small angular extents on the sky, and the emission may be dominated by a central AGN or a cool bright X-ray core. 
The goal of the project described in this paper was, therefore, to search for distant X-ray extended sources that do not appear extended in Rosat PSPC data (due to instrument limitations), as used by the SHARC surveys.

As distant $(\gtrsim 0.8) \mathrm{X}$-ray luminous clusters are rare, it is necessary to search the largest possible region of the sky for which deep X-ray exposures are available. We therefore used our previously reported Bright-SHARC survey (e.g. Romer et al. 2000). From the entire catalog of sources detected in the ROSAT pointed observations (covering about $180 \mathrm{deg}^{2}$; the effective area decreases with the sensitivity level, see Sect. 4), we removed:

- all the sources detected as extended in the PSPC data above $1.5 \times 10^{-13} \mathrm{erg} \mathrm{cm} \mathrm{cm}^{-2} \mathrm{~s}^{-1}$ found in the original BrightSHARC survey (Romer et al. 2000); here our survey goes almost 10 times fainter and is therefore limited by definition to sources not appearing as extended;

- all sources with a detection limit lower than $3.5 \sigma$;

- all the sources identified with known single objects (single galaxies, QSOs and stars) from the NED and Simbad databases;

- all the sources with a clear NVSS (Dickey \& Lockman 1990) radio source, to avoid as much as possible bright distant AGNs or QSOs, within a radius of 2 arcmin. We also checked a posteriori that no radio source from the Radio Master Catalog available at http://heasarc.gsfc.nasa.gov/ was detected within our X-ray contours. More specifically, among the 38 entries of Table 1, 35 are covered by the FIRST radio survey (White et al. 1997), but no radio source is located within our X-ray contours.

- all the sources with a clearly visible optical object in the DSS photographic plates (Lasker et al. 1990) within a radius of 2 arcmin.

We finally distilled down the original list of over 3000 objects to 36 candidates that we are in the process of systematically examining with new deep optical imaging, optical spectroscopy, and X-ray followup observations when possible. We also verified there was no duplication between our source list and what was available in the literature for these targets up to Nov. 2006. These 36 remaining sources are then:

- either rich clusters, too distant and/or too faint and/or too far offaxis to be detected as extended in our automated processing scheme. As seen in the images, however, a few objects show possible evidence for extended X-ray emission. These candidate clusters are also too distant to have their galaxy population clearly visible in the DSS. Assuming a magnitude of $M_{R}=-23$ for a typical brightest cluster galaxy, the cosmological parameters given at the end of this section and assuming a magnitude limit of $R \sim 18$ for the DSS, this gives a minimal redshift of 0.3 for these clusters (and lower if we assume they are groups instead of clusters, see the text for individual objects below);

- either distant X-ray active objects (e.g. AGNs, QSOs) or stars too faint to be seen in the DSS.

Although the project is not complete, we have gathered a significant amount of data that warrant a "mid-term" report. It is interesting to compare these results with newer ongoing surveys such as ChaMP (Barkhouse et al. 2006) so that when designing future missions devoted to large sky surveys it can be judged whether it is better to design a telescope that cuts off at relatively low energies (similar to ROSAT) versus a smaller field of view telescope but with significant collecting area up to at least $7 \mathrm{keV}$ (such as Chandra or XMM-Newton).
It is also important to note how followup ground based observations are beginning to reveal sets of underlumnious X-ray clusters which have the potential of complicating cosmological interpretations of $\mathrm{S}-\mathrm{Z}$ and $\mathrm{X}$-ray cluster surveys.

We will assume for the purpose of calculations that $H_{0}=$ $71 \mathrm{~km} \mathrm{~s}^{-1} \mathrm{Mpc}^{-1}, \Omega_{\Lambda}=0.73$ and $\Omega_{\mathrm{m}}=0.27$.

\section{The data}

We give the list of our 36 candidates in Table 1 . This table summarizes for each object the observational details described hereafter.

\subsection{Optical imaging}

We first observed deep $i^{\prime}$ (and sometimes $r^{\prime}$ ) images of 13 candidates at the ARC $3.5 \mathrm{~m}$ telescope with SPIcam ${ }^{1}$. Exposure times ranged from 20 to $90 \mathrm{~min}$. Two other candidates were observed in the $R$ band at the ESO $3.5 \mathrm{~m}$ telescope with EFOSC2 with exposure times of $10 \mathrm{~min}$. Three candidates were observed in the $R$ band at the OHP $1.2 \mathrm{~m}$ telescope and CCD camera with exposure times between 40 and $150 \mathrm{~min}$. We obtained $B, V$ and $R$ band observations for one candidate at the CFHT with the CFH12K camera ( $B: 20 \mathrm{~min}, V: 15 \mathrm{~min}, R: 10 \mathrm{~min}$ ). Finally, we observed one candidate with the Gemini north telescope in $r^{\prime}$ and $i^{\prime}$ for 15 and $17 \mathrm{~min}$, respectively.

For the 16 remaining candidates, 13 are covered by the SDSS and 3 by the DSS Red and Blue photographic plates (McLean et al. 2000). Although the SDSS and DSS data are shallower than our direct observations, by quadratically summing the data in all available bands $\left(u, g^{\prime}, r^{\prime}, i^{\prime}, z^{\prime}\right.$ for SDSS, and blue and red for DSS), taking into account the typical noise in each band, we then produced deeper images than those in the individual bands. This method is not rigourously optimal to detect the faintest possible objects (see e.g. Szalay et al. 1999, for a better but more complex extraction method) and is not intended to product any calibrated magnitudes. We used this method because our goal was to get a deep representation of the field object populations, in order to decide which target should be followed with very deep images. The up-to-date results of the optical imaging along with the superposed X-ray contours are given in the on-line material. The images made by superposing various bands will be refered to hereafter as " $\chi$ " images.

\subsection{X-ray imaging}

The target selection was made on the basis of ROSAT PSPC images treated by a automated procedure described in Romer et al. (2000). In addition, we found in the Chandra and $X M M$-Newton archives X-ray data for 6 candidates (5 candidates with XMM-Newton data and 1 with Chandra data), but often at the field edge, with low exposure times, or both. The images in the Appendix were overlayed with XMM/Chandra data when available and with ROSAT PSPC data if not. X-ray contours were computed for each candidate at $1 \sigma$ intervals starting from the $3 \sigma$ level. These levels were computed using the background estimated from the count rate in an arbitrarily empty region close to the X-ray source. The ROSAT PSPC images were smoothed over a 1.5 arcmin Gaussian window prior to generating the contours.

\footnotetext{
${ }^{1}$ See http://www. apo.nmsu. edu for details.
} 
Table 1. 36 X-ray sources (plus two additional structures) in the survey. We give the coordinates (from the SHARC wavelet analysis, see Adami et al. 2000), the optical imaging data origin and characteristics (exposure time ET in minutes), the X-ray data (ROSAT PSPC, XMM or Chandra and radial offset in arcmin), the optical spectroscopy data origin (S for SDSS, CA for Calar Alto or V for the VIMOS IFU), the source status (Single: X-ray point source, Cluster: galaxy structure, Pending: to be determined), an estimated redshift (if a galaxy structure), the ROSAT PSPC

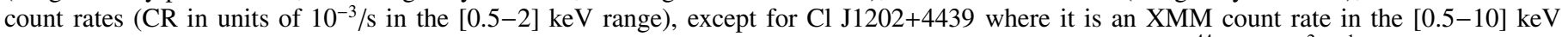
band; all the luminosities given are based on the assumption of the maximum estimated redshift, in units of $10^{44} \mathrm{erg} \mathrm{cm}^{-2} \mathrm{~s}^{-1}$, and $n_{\mathrm{H}}$ in units of $10^{22} \mathrm{~cm}^{-2}$. The two additional sources are not detected in the original SHARC analysis and we therefore do not give X-ray properties for them.

\begin{tabular}{|c|c|c|c|c|c|c|c|c|c|c|}
\hline Source & $\overline{\alpha(2000)}$ & $\overline{\delta(2000)}$ & Imaging & $\overline{E T}$ & $\overline{X-\text {-rays }}$ & Sp & $\overline{\text { Status }}$ & $\bar{z}$ & $\overline{\mathrm{CR}}$ & $\overline{L_{\mathrm{Xbol}} / n_{\mathrm{H}}}$ \\
\hline Cl0223-0856 & 022306.10 & -085647.6 & OHP $1.2 \mathrm{~m} R$ & 60 & ROSAT 8 & S & Cluster? & $0.49 ?$ & 1.97 & $0.424 / .029$ \\
\hline Cl0240-0801 & 024009.56 & -080106.6 & $\operatorname{SDSS} \chi^{2}$ & & ROSAT 19 & & Pending & & 3.08 & \\
\hline Cl0241-0802 & 024103.56 & -080211.3 & $\mathrm{ESO} 3.5 \mathrm{~m} \mathrm{I}$ & 10 & ROSAT 12 & & Cluster? & $0.55 ?$ & 2.71 & $0.762 / .035$ \\
\hline Cl0242-0756 & 024222.17 & -075604.9 & $\operatorname{SDSS} \chi^{2}$ & & ROSAT 27 & & Single? & & 7.98 & \\
\hline $\mathrm{Cl0254+0012}$ & 025424.25 & +00 1252.6 & DSSRed/Blue $\chi^{2}$ & & ROSAT 11 & $\mathrm{~S}$ & Pending & & 3.23 & \\
\hline Cl0302-1526 & 030241.77 & -152647.0 & DSSRed/Blue $\chi^{2}$ & & ROSAT 17 & & Single? & & 2.25 & \\
\hline Cl0317-0259 & 031726.87 & -025934.5 & DSSRed/Blue $\chi^{2}$ & & $\begin{array}{l}\text { ROSAT } 8 \\
\text { XCS }\end{array}$ & & Single? & & 1.89 & \\
\hline $\mathrm{Cl} 0413+1215$ & 041354.03 & +121558.8 & $\mathrm{OHP} 1.2 \mathrm{~m} R$ & 150 & ROSAT 13 & & Pending & & 2.43 & \\
\hline $\mathrm{Cl} 0922+6217$ & 092253.19 & +621714.8 & $\mathrm{OHP} 1.2 \mathrm{~m} R$ & 40 & ROSAT 9 & & Pending & & 2.73 & \\
\hline $\mathrm{Cl} 10937+6105$ & 093748.47 & +610527.6 & ARC $3.5 \mathrm{mi} i^{\prime}$ & 36 & ROSAT 20 & & Pending & & 3.10 & \\
\hline Cl J1024+1935 & 102423.89 & +193515.8 & ARC $3.5 \mathrm{~m} i^{\prime}$ & 50 & ROSAT 20 & & Cluster? & $0.15-0.65 ?$ & 6.09 & $2.49 / .0214$ \\
\hline Cl J1024+1943 & 102437.92 & +194314.9 & ARC $3.5 \mathrm{~m} i^{\prime}$ & 90 & ROSAT 17 & & Pending & & 3.10 & \\
\hline Cl J1050+6317 & 105017.65 & +631745.2 & ARC $3.5 \mathrm{~m} i^{\prime}$ & 50 & ROSAT 25 & & Pending & & 4.08 & \\
\hline Cl J1052+5655 & 105211.89 & +565535.3 & $\operatorname{SDSS} \chi^{2}$ & & ROSAT 26 & $\mathrm{~S}$ & Single? & & 10.69 & \\
\hline $\mathrm{Cl} \mathrm{J} 1052+5400$ & 105246.60 & +540002.6 & ARC $3.5 \mathrm{mi} i^{\prime}$ & 90 & XMM 12 & & Spurious? & & 2.02 & \\
\hline $\mathrm{Cl} \mathrm{J} 1102+2514$ & 110208.95 & +251418.5 & ARC $3.5 \mathrm{mi}$ & 50 & ROSAT 12 & & Cluster? & $0.15-0.65 ?$ & 3.65 & $1.24 / 0.0140$ \\
\hline Cl J1103+2458 & 110321.80 & +245849.8 & $\operatorname{SDSS} \chi^{2}$ & & ROSAT 11 & & Pending & & 1.31 & \\
\hline $\mathrm{Cl} \mathrm{J} 1113+4042$ & 111334.59 & +404232.9 & ARC $3.5 \mathrm{~m} i^{\prime}$ & 90 & Chandra 13 & CA & Cluster & 0.51 & 4.68 & $1.09 / 0.0184$ \\
\hline Cl J1120+1254 & 112048.59 & +125458.8 & $\begin{array}{l}\text { CFHT } 3.6 \mathrm{~m} B \\
\text { CFHT } 3.6 \mathrm{~m} V \\
\text { CFHT } 3.6 \mathrm{~m} R\end{array}$ & $\begin{array}{l}20 \\
15 \\
10\end{array}$ & $\begin{array}{c}\text { XMM } 9 \\
\text { XCS }\end{array}$ & & Single & & 3.53 & \\
\hline $\mathrm{Cl} \mathrm{J} 1121+4309$ & 112140.67 & +430906.8 & $\operatorname{SDSS} x^{2}$ & & ROSAT 9 & & Pending & & 3.14 & \\
\hline $\mathrm{Cl} \mathrm{J} 1121+0338$ & 112156.65 & +033818.8 & $\begin{array}{c}\text { ARC } 3.5 \mathrm{~m} r^{\prime} \\
\text { ARC } 3.5 \mathrm{~m} i^{\prime} \text { band }\end{array}$ & $\begin{array}{l}90 \\
80\end{array}$ & ROSAT 30 & $\mathrm{~S} / \mathrm{V}$ & Single & & 15.18 & \\
\hline $\mathrm{Cl} \mathrm{J} 1158+5541$ & 115850.65 & +554134.4 & $\operatorname{SDSS} \chi^{2}$ & & XMM 12 & & Pending & & 1.92 & \\
\hline $\mathrm{Cl} \mathrm{J1202+4439}$ & 120233.03 & +443942.8 & $\operatorname{SDSS} \chi^{2}$ & & $\begin{array}{l}\text { XMM } 10 \\
\text { XCS }\end{array}$ & & Cluster? & 0.28 & 7.36 & $0.212 / .0135$ \\
\hline $\mathrm{Cl} \mathrm{J} 1207+4429$ & 120740.91 & +442938.8 & $\operatorname{SDSS} \chi^{2}$ & & ROSAT 12 & & Pending & & 1.97 & \\
\hline Cl J1213+3908 & 121332.88 & +390824.7 & ARC $3.5 \mathrm{mi}$ & 30 & ROSAT 24 & & Single? & & 7.47 & \\
\hline $\mathrm{Cl} \mathrm{J} 1213+3317$ & 121353.75 & +331727.4 & $\operatorname{SDSS} \chi^{2}$ & & ROSAT 16 & & Pending & & 2.46 & \\
\hline Cl J1214+1254 & 121450.32 & +125401.9 & ESO $3.5 \mathrm{~m} \mathrm{I}$ & 10 & ROSAT 14 & & Pending & & 6.15 & \\
\hline $\mathrm{Cl} \mathrm{J} 1216+3318$ & 121622.89 & +3318 28.5 & ARC $3.5 \mathrm{~m} r^{\prime}$ & 90 & ROSAT 17 & & Pending & & 7.82 & \\
\hline Cl J1216+3318 & & & ARC $3.5 \mathrm{~m} i^{\prime}$ band & 90 & & & & & & \\
\hline Cl J1234+3755 & 123400.88 & +375549.2 & $\operatorname{SDSS} \chi^{2}$ & & ROSAT 20 & $\mathrm{~S}$ & Single & & 4.65 & \\
\hline $\mathrm{Cl} \mathrm{J} 1237+2800$ & 123718.90 & +280016.5 & $\operatorname{SDSS} \chi^{2}$ & & ROSAT 18 & & Pending & & 5.21 & \\
\hline $\mathrm{Cl} \mathrm{J} 1259+2547$ & 125920.71 & +254710.4 & $\operatorname{SDSS} \chi^{2}$ & & ROSAT 51 & & Pending & & 3.15 & \\
\hline Cl J1343+2716 & 134308.32 & +271638.7 & $\operatorname{SDSS} \chi^{2}$ & & ROSAT 13 & & Pending & & 2.63 & \\
\hline $\mathrm{Cl} \mathrm{J} 1350+6028$ & 135045.95 & +602839.2 & ARC $3.5 \mathrm{~m} \mathrm{i}$ & 50 & ROSAT 21 & & Single? & & 4.01 & \\
\hline Cl J1411+5933 & 141108.37 & +593312.5 & ARC $3.5 \mathrm{~m} i^{\prime}$ & 50 & ROSAT 25 & & Cluster? & $0.25-1 ?$ & 5.30 & $5.97 / 0.0166$ \\
\hline Cl J1514+4351 & 151411.33 & +435123.9 & ARC $3.5 \mathrm{~m} i^{\prime}$ & 20 & ROSAT 12 & $\mathrm{~S}$ & Cluster? & $0.3-1 ?$ & 2.58 & 2.90 \\
\hline Cl J1651+6107 & 165102.95 & +610725.3 & $\begin{array}{l}\text { Gemini } 8.2 \mathrm{~m} r^{\prime} \\
\text { Gemini } 8.2 \mathrm{~m} i^{\prime}\end{array}$ & $\begin{array}{l}15 \\
17\end{array}$ & XMM 9 & CA & Cluster? & $0.2-0.5 ?$ & 2.72 & $0.608 / .025$ \\
\hline & 105030 & +631918 & ARC $3.5 \mathrm{~m} i^{\prime}$ band & 50 & ROSAT & $\mathrm{CA}$ & Cluster & 0.53 & & \\
\hline & 111342 & +404222 & ARC $3.5 \mathrm{~m} i^{\prime}$ band & 90 & ROSAT & & Cluster? & $0.15-0.6 ?$ & & \\
\hline
\end{tabular}

\subsection{Spectroscopy}

We checked the NED database for available redshifts in the considered areas (at this writing). We also obtained single slit spectra at the Calar Alto $3.5 \mathrm{~m}$ telescope with the MOSCA spectrograph $^{2}$ for three candidates (exposure times ranging between 30 min and 3 h). Finally, we got a 12 h VIMOS IFU (Le Fèvre et al. 2003) ${ }^{3}$ observation of one candidate (but weather conditions were quite poor).

\footnotetext{
${ }^{2}$ See http://w3.caha.es/CAHA/Instruments/MOSCA/ manual.html

${ }^{3}$ http://www . eso.org/instruments/vimos/
}

\section{Description of the 36 candidates}

Here we discuss the possible nature of each candidate with the data we have in hand. Our conclusions are summarized in Table 1 and the corresponding figures are given in the on-line appendix.

When objects are visible in the optical images (excluding the $\chi^{2}$ images) within the X-ray contours, we used the brightest one to compute a minimal redshift for the structure, assuming that this object is the dominant galaxy of the structure. We assumed an absolute magnitude of $M_{R}=-23$ and $M_{i^{\prime}}=-23.5$ for the 
dominant galaxy in each structure (these values are typical in nearby clusters).

We also computed an X-ray flux in the [0.5-2] keV energy range using WEBPIMMS and WEBSPEC, as well as $L_{\mathrm{Xbol}}$ (using XSPEC) for the X-ray objects that we considered as possible clusters. We assumed a mean $k T$ of $3 \mathrm{keV}$ (the effect of the assumed $k T$ over a reasonable temperature range of $2-10 \mathrm{keV}$ is, however, less than 10\%) and a Mekal model, with a fixed metallicity $Z=0.3 Z_{\odot}$. We used the hydrogen column density in the Galaxy for each relevant pointing (see Table 1).

\subsection{ARC imaging}

Thirteen candidates have been followed up with imaging at the ARC $3.5 \mathrm{~m}$ telescope at least in the $i^{\prime}$ band.

Cl J0937+6105 and Cl J1050+6317: the central X-ray contours of $\mathrm{Cl} \mathrm{J0937+6105}$ and $\mathrm{Cl} \mathrm{J1050+6317} \mathrm{are} \mathrm{not} \mathrm{clearly} \mathrm{as-}$ sociated with any optical object.

Cl J1024+1935 has a regular shape but we have no spectroscopy for this candidate. The brightest optical object has a magnitude of $i^{\prime}=19.4$ and is possibly a quasar or an AGN simi-

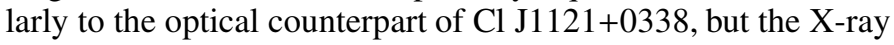
countours can be typical of a nearby galaxy structure.

Cl J1024+1943 is an X-ray source associated with a complex optical object population, that consists of both quite bright and very faint objects.

Cl J1052+5400: this field contains a rich galaxy population. However, the ROSAT PSPC X-ray source was not detected in our analysis of recent XMM-Newton data. This source could be a variable point source, as the image quality is not good enough to determine if the source is extended. Since it is not present in the $X M M-N e w t o n$ data, the source could also be spurious.

Cl $\mathbf{J 1 1 0 2 + 2 5 1 4}$ is a relatively strong X-ray source that is well centered on a relatively bright non circular optical object $\left(i^{\prime}=19.2\right)$ and the contours also include several other optical counterparts. This X-ray source is probably a group or a cluster between redshifts $z \sim 0.15$ and $\sim 0.65$.

Cl J1113+4042 is a complex X-ray source also observed with Chandra. The X-ray data are not deep enough and too far off axis (13', Chandra $14^{\prime}$, ROSAT) to allow spectral investigations or to determine a statistically significant X-ray extent. The area probably includes an AGN (the west source) and two real galaxy structures (see Sect. 3.8 for a discussion of the east structure). We determined three redshifts close to the central X-ray emission. Two of the main galaxies are probably associated with the X-ray emission, are at a redshift of $\sim 0.5$ and have an early

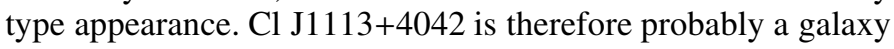
group at $z \sim 0.5$.

Cl J1121+0338 is a relatively strong X-ray source (two times stronger than $\mathrm{Cl} \mathrm{J1024+1935)} \mathrm{and} \mathrm{is} \mathrm{well} \mathrm{centered} \mathrm{on} \mathrm{a}$ QSO observed by the SDSS at $z=0.839$. We also obtained VIMOS IFU spectroscopic data for this region. No galaxy concentration appears in redshift space and we therefore conclude

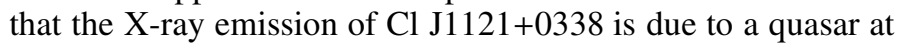
$z \sim 0.84$.

Cl J1213+3908 and Cl J1350+6028 have outer X-ray contours that include several peaks (the X-ray emission of Cl J1213+3908 covers the whole optical field of view) or are very large. The optical fields are dense enough, however, that the coincidence between these inner contour peaks and the optical objects could be purely by chance.

Cl J1216+3318 has a rich galaxy population that extends outside the border of the X-ray contours. The X-ray contours are quite irregular and it is difficult to conclude with the data in

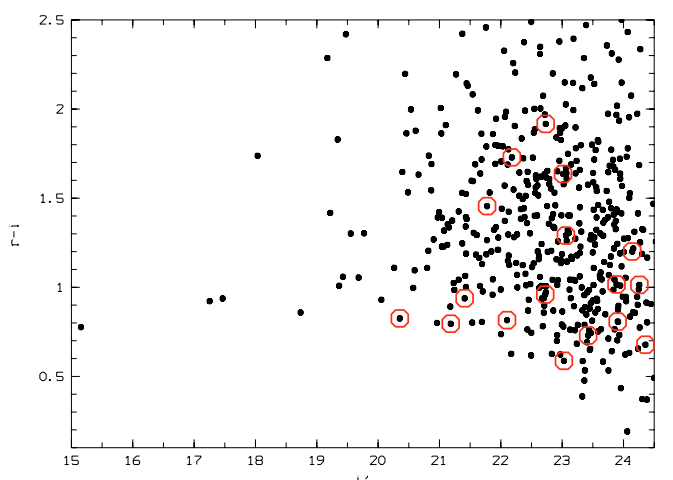

Fig. 1. $r^{\prime}-i^{\prime}$ vs. $i^{\prime}$ color magnitude relation for the $\mathrm{Cl} \mathrm{J} 1216+3318$ field of view. The larger (red) filled circles are the galaxies included within the X-ray contours.

hand whether this a cluster or a single source. In order to explore further the possible existence of a cluster we have plotted in Fig. 1 the galaxy color magnitude relation in the field. The large (red) circles are the optical objects within the X-ray contours. When we also include objects outside the X-ray contours, we find marginal evidence for a red sequence around $r^{\prime}-i^{\prime}=$ 0.8 (11 objects among the 17 within the X-ray contours have $\left.0.5<r^{\prime}-i^{\prime}<1.1\right)$, that would place a galaxy structure between $z=0.2$ and 0.5 (from Fukugita et al. 1995). However, the absence of a central bright galaxy makes it difficult to conclude if

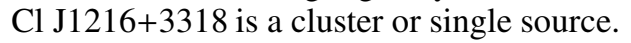

Cl J1411+5933 is an X-ray source with at least 5 optical objects within the X-ray contours, the brightest one having an $i^{\prime}$ magnitude of 20.7. This would place the galaxy structure at $z \sim 1$ if it is an $M_{i^{\prime}}=-23.5$ galaxy associated to a rich cluster or at $z \sim 0.25$ if it is an $M_{i^{\prime}}=-20$ group central galaxy.

Cl J1514+4351: This X-ray source is quite elongated, hence it is apparently an extended X-ray source. Several optical objects (including at least two galaxies) are visible within the X-ray contours. The brightest (and most extended one) has an $i^{\prime}$ magnitude of 20.9. This apparent magnitude places this cluster candidate at $z \sim 1$ if this is an $M_{i^{\prime}}=-23.5$ galaxy associated with a rich cluster, or at $z \sim 0.3$ if this is an $M_{i^{\prime}}=-20$ central galaxy of a group. A foreground galaxy has been measured by the SDSS at $z=$ 0.16518 and is associated with a larger foreground galaxy cluster at this redshift. However, this foreground structure is probably not related to the X-ray source. This is because the X-ray emission does not overlap the $z \sim 0.16$ galaxy.

\subsection{Gemini data for $\mathrm{Cl} J 1651+6107$}

This candidate has a relatively low Galactic latitude $\left(\sim 37^{\circ}\right)$ and is located in a region populated by Galactic stars and with a prominent galactic $\mathrm{H} \alpha$ emission coming from the Draco cloud (e.g. Penprase et al. 2000). This is confirmed by the two spectra of cold stars (located at the upper left and lower right in Fig. A.36 of the online data) we obtained at Calar Alto (with MOSCA)

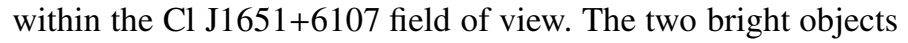
embedded in the diffuse optical emission (see Fig. A.36 in the Appendix) are also stars, as deduced from the $r^{\prime}$ Gemini image.

We found XMM data in the archive for this candidate, but the exposure time is far too low to allow any spectral analysis. The X-ray emission is, however, located on the top of 7 very faint objects that are galaxies, based on the imaging data used in Figs. 2-4. 


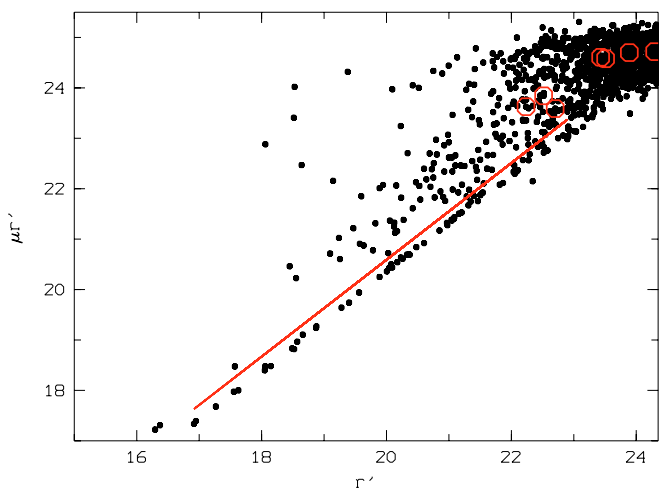

Fig. 2. Cl J1651+6107: central surface brightness versus total $r^{\prime}$ magnitude diagram used to distinguish stars from galaxies, see text.

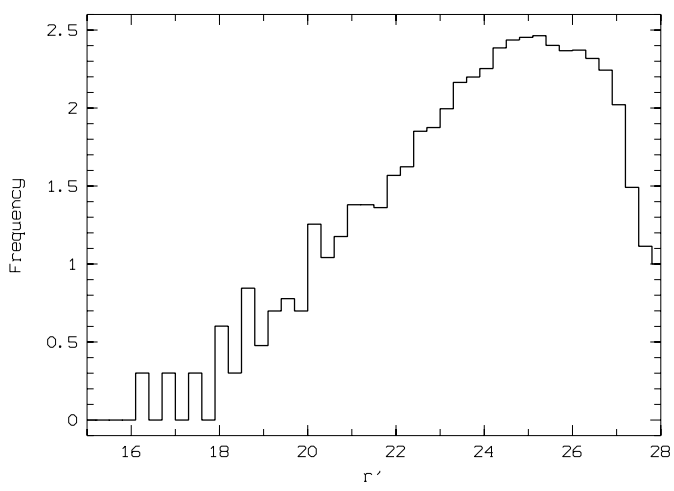

Fig. 3. Log-normal $\mathrm{Cl} \mathrm{J} 1651+6107 r^{\prime}$ magnitude histogram for the Gemini field, suggesting completeness up to $r^{\prime} \sim 24.5$.

Figure 2 shows a clear star-galaxy separation down to $r^{\prime} \sim$ 22.9. We considered all objects fainter than $r^{\prime} \sim 22.9$ as galaxies, since the Galactic star contribution at these magnitudes is very low (e.g. Adami et al. 2006a).

These results suggest that we have found a structure of galaxies. In order to estimate its redshift, we limited our analysis of the $r^{\prime}$ data to $r^{\prime}=24.5$ (as suggested by Fig. 3) and we plotted in Fig. 4 the color magnitude relation of all objects classified as galaxies. The three brightest galaxies within the X-ray contours have $r^{\prime}-i^{\prime}$ colors close to 0.4. Following Fukugita et al. (1995) and assuming these are early type galaxies, this would place the structure between $z=0.2$ and 0.5 . The fainter objects are probably very low mass objects which were not able to retain most of their metals and appear therefore quite blue (e.g. Adami et al. 2006b).

Given the magnitude of the brightest galaxy within the X-ray contours one interpretation is that this structure is a group, since this magnitude is too faint to be a cluster dominant galaxy at $z \leq$ 0.5 . The absolute $r^{\prime}$ magnitude of the brightest galaxy would be -17.7 at $z=0.2$ and -20.0 at $z=0.5$. The absolute magnitudes are in the range of $L^{*}$ values for groups or clusters. An alternative explanation is that $\mathrm{Cl} \mathrm{J} 1651+6107$ is a cluster at $z \sim 1$ if our interpretation of the colors did not produce the true value of the redshift.

\subsection{CFHT data for Cl J1120+1254}

We have obtained $B, V$ and $R$ CFHT CFH12K images for Cl J1120+1254. The images show several objects inside the $\mathrm{X}$-ray contours. One of the two brightest galaxies has a very blue color, the other a very red color (Fig. 5).

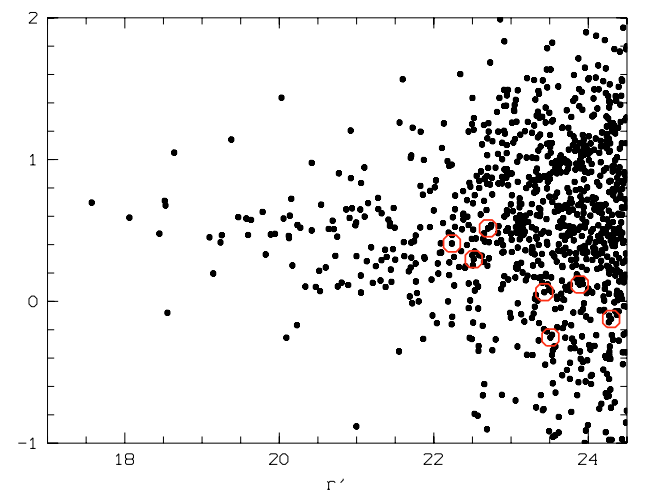

Fig. 4. Cl J1651+6107: $r^{\prime}-i^{\prime}$ color versus $r^{\prime}$ magnitude for objects classified as galaxies. The 7 large (red) circles correspond to the galaxies within the X-ray contours.

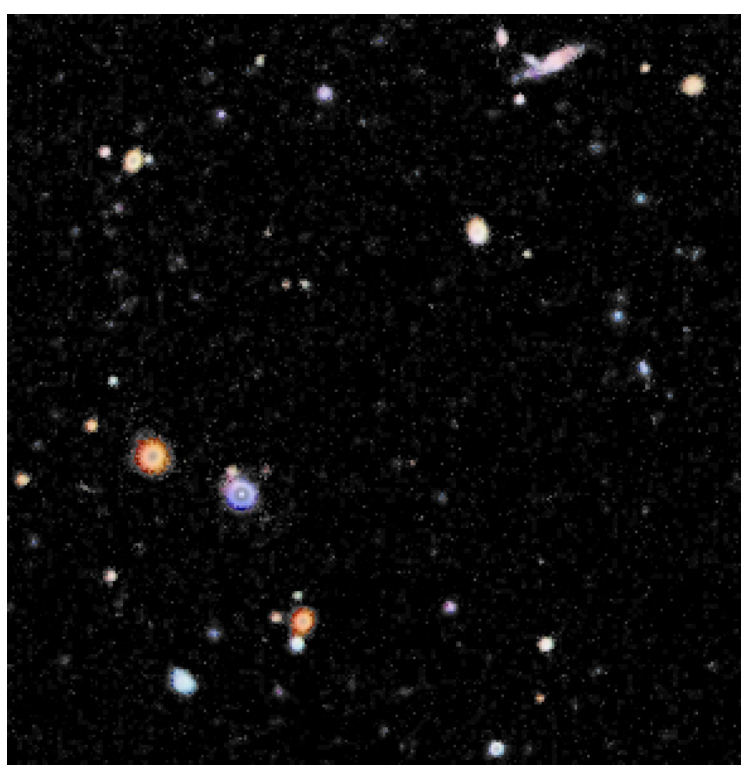

Fig. 5. $B V R$ color image of $\mathrm{Cl} \mathrm{J} 1120+1254$. The two bright objects at the image bottom (one blue, one red) are the brightest objects within the $\mathrm{X}$-ray contours.

There are XMM data available for this candidate but the source is located off axis $\left(\sim 9^{\prime}\right)$, so the angular resolution is degraded by about $50 \%$ (this XMM source is however detected as not extended by the ongoing XCS survey, Romer et al., private communication and 2001). For display purposes, we made two images, one in the [0.5-2.0] keV band (soft) and the other in the [2.0-10.0] keV band (hard). Clusters are expected to appear stronger in the soft band than in the hard band, while AGN should look point-like in both bands. Following this, we have plotted in Fig. 6 both the Cl J1120+1254 X-ray source and the X-ray image of a known galaxy structure (ClG J1205+4429, hereafter Cl J1205, see Ulmer et al. 2005). The image of this known galaxy structure also has a very prominent AGN in its field (at the north east in the image). Cl J1205 (the known galaxy structure) is a strong source in the soft band and a very weak one in the hard band, while the AGN can be seen to be relatively strong in both bands (although weaker in the soft band than in

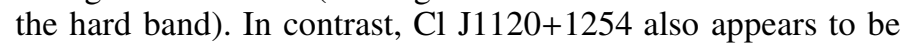
relatively strong in both bands (even if weaker in the hard band compared to the soft band). Given its X-ray image, however, we conclude that $\mathrm{Cl} \mathrm{J} 1120+1254$ is probably an X-ray point source, 


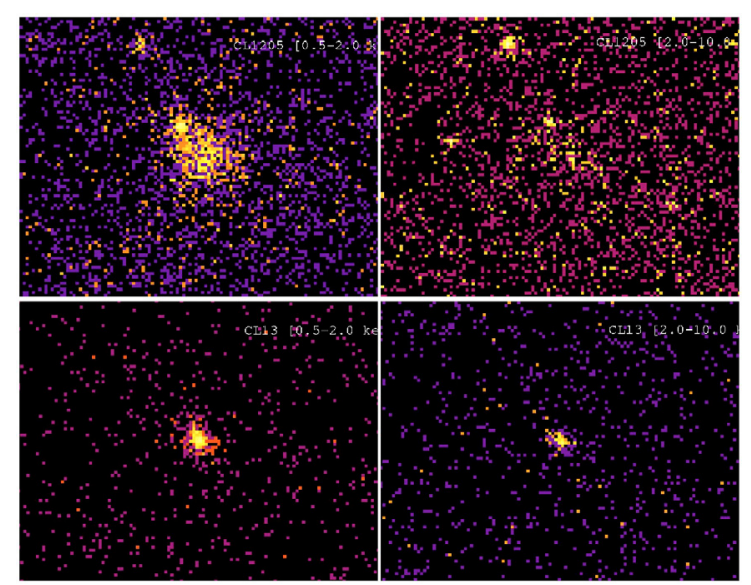

Fig. 6. A known galaxy structure, ClG J1205+4429 (top) with a line-ofsight X-ray detectable AGN in the upper left of the image, see Ulmer et al. (2005). [0.5-2.0] keV (left) and [2.0-10.0] keV band (right). Similar images of Cl J1120+1254 (bottom).

despite that the spectral shape is not the same as that of the AGN in the field of Cl J1205.

\subsection{ESO 3.5 m EFOSC2 data for Cl J0241-0802 and $\mathrm{Cl} \mathrm{J1214+1254}$}

Cl J0241-0802 and Cl J1214+1254 have been imaged with the ESO $3.5 \mathrm{~m}$ telescope and the EFOSC2 instrument (imaging mode). Both candidates are associated with relatively bright optical objects.

Cl J0241-0802 is probably a galaxy structure given the large number of optical sources and the "dominant galaxy" appearance of the brightest object visible within the X-ray contours. This object has an I magnitude of 19, placing the possible galaxy structure at $z \sim 0.55$. The $\mathrm{X}$-ray contours also suggest that this is an extended X-ray source. At such a redshift, its extent corresponds to a diameter of $500 \mathrm{kpc}$, in good agreement with the extent of a typical group of galaxies (or poor cluster).

Cl J1214+1254 is poorer than Cl J0241-0802 from an optical point of view but its X-ray shape seems extended. It is however impossible to conclude on the nature of this source with the data in hand.

\subsection{OHP data for Cl J0223-0856, Cl J0413+1215 and $\mathrm{Cl} \mathrm{J} 0922+6217$}

These three cluster candidates were observed at the OHP $1.2 \mathrm{~m}$ telescope.

The ROSAT X-ray images of $\mathrm{Cl}$ J0223-0856 and $\mathrm{Cl} \mathrm{J} 0413+1215$ are relatively round.

In the $R$ image of $\mathbf{C l ~} \mathbf{J 0 2 2 3 - 0 8 5 6}$ there are four faint objects visible within the X-ray contours (see image in online data Fig. 1 and magnitude histogram in Fig. 7). If Cl J0223-0856 is a cluster of galaxies, then its redshift could be $z=0.49$ based on the brightest detected galaxy. The angular extent of the X-ray emission is PSF dominated but is equivalent to a $200 \mathrm{kpc}$ diameter circle at this redshift. This is compatible with its being a cluster core or a group. C10223-0856 could therefore be a galaxy structure.

There is only one optical object visible within the X-ray contours of $\mathbf{C l ~} \mathbf{J 0 4 1 3 + 1 2 1 5}$, but the source could be a cluster of galaxies as the magnitude limit of the $R$ band image is about 21

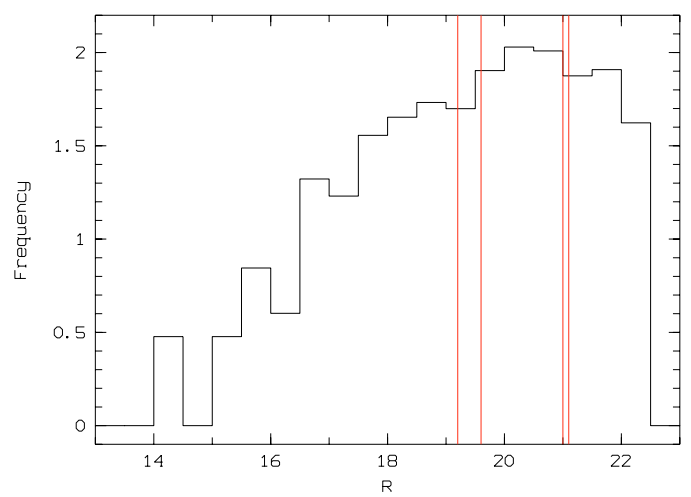

Fig. 7. Log-normal Cl J0223-0856 $R$ magnitude histogram. The vertical (red) lines are the four objects detected within the X-ray contours.

which means it is unlikely that fainter cluster member galaxies would be visible in the $R$ image. If $\mathrm{Cl} \mathrm{J0413+1215}$ is indeed a cluster of galaxies, its redshift could be $z \sim 1$ based only on the single detected galaxy $(R=21.7)$ within the X-ray contours. The size of the X-ray emission is also PSF dominated and is equivalent to a $300 \mathrm{kpc}$ diameter circle at this redshift. The data are, however, too sparse to allow us to make a definitive statement about its nature.

Cl J0922+6217 has faint optical objects within its contours. The X-ray source could be either a cluster of galaxies or an X-ray point source.

\subsection{SDSS data}

Thirteen of our cluster candidates have no deep CCD imaging. For each of these objects, we summed in quadrature all the SDSS available bands $\left(u, g^{\prime}, r^{\prime}, i^{\prime}, z^{\prime}\right)$ to make a visible band image onto which to overlay the X-ray contours.

Cl J0240-0801, Cl J1103+2458, Cl J1207+4429 and Cl J1343+2716 all have faint optical objects within their contours. These X-ray sources could be either clusters of galaxies or X-ray point sources.

Cl J1052 $+\mathbf{5 6 5 5}$ is possibly made up of a collection of X-ray point sources. Within this region one galaxy has a measured redshift of $z=0.52147$ (taken from NED), but it falls at the border of the X-ray contours. The data are too noisy to determine if the $\mathrm{X}$-ray source is truly extended or not. It is probably a collection of individual sources.

Cl J1121+4309 has a quite regular and PSF dominated $\mathrm{X}$-ray shape. There is no visible object within ROSAT PSPC $\mathrm{X}$-ray contours. The optical counterpart is very faint.

Cl J1158+5541 has XMM data, but the image is located at the edge of the MOS fields ( $\sim 12^{\prime}$ off axis) and is not in the PN field. This prevents us from deriving an X-ray spectrum. The PSF is badly degraded at this location and we cannot provide a reliable measure of the extent of this X-ray source either. This candidate is however associated with a faint optical object population, and thus its nature is indeterminate between a distant cluster and an AGN.

Cl $\mathbf{J 1 2 0 2 + 4 4 3 9}$ is a relatively strong $X$-ray source $(S / N$ greater than 6 in the ROSAT PSPC data) for which $X M M$-Newton data (net exposure time of $36.7 \mathrm{ks}$ after flare removal) are also available. This source was not detected as extended by the ongoing XCS survey (Romer et al.: private communication and 2001). Our XMM-Newton analysis generated $\sim 300$ photons in the source after background removal; we produced an X-ray spectrum (Fig. 8) and fit a MEKAL model 


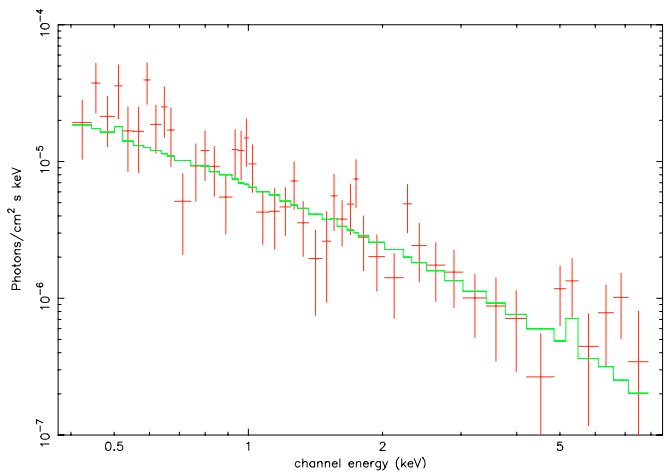

Fig. 8. X-ray photon spectrum of Cl J1202+4439.

$\left(N_{\mathrm{H}}=1.35 \times 10^{20} \mathrm{~cm}^{-2}\right.$ and fixed metallicity of $\left.0.3 Z_{\odot}\right)$. The best fit was obtained for a redshift of $0.28_{-0.28}^{+0.21}$. The spectrum in Fig. 8 shows a $\sim 1 \sigma$ feature that could be due to Fe emission at about $5.2 \mathrm{keV}$, but many other similarly sized features exist in the spectrum. The $5.2 \mathrm{keV}$ energy for the rest frame $6.7 \mathrm{keV}$ Fe line is consistent with the derived redshift. There are two optical objects within the X-ray contours which have magnitudes consistent with being galaxies at this $\sim 0.3$ redshift. The object is then possibly a group of galaxies at $z \sim 0.3$ with an estimated luminosity of $1.1 \times 10^{43} \mathrm{erg} / \mathrm{s}$ (in the [0.5-10] keV range) but the nature of the structure is still to be confirmed with optical spectroscopy. This would be typical of a bright galaxy group (e.g. Jones et al. 2003).

Cl J1213+3317 and Cl J1237+2800 consist of several large $\mathrm{X}$-ray sources with embedded faint optical objects. These candidates could be either clusters of galaxies or made of several unrelated X-ray point sources.

Cl J1234+3755 is possibly an extended X-ray source. There is an SDSS QSO on the edge of the X-ray contours (at $z=$ 0.57313 ), so some of the X-ray emission could originate from this QSO. We cannot exclude the possibility of having a QSO embedded in a cluster.

Cl J0242-0756 and Cl J1259+2547 are made up of weak $\mathrm{X}$-ray sources with a few faint optical objects within their contours. Cl J1259+2547 seems more extended than Cl J0242-0756.

\subsection{DSS2 red and blue data for Cl J0254+0012, $\mathrm{Cl}$ J0302-1526 and Cl J0317-0259}

These 3 candidates have no CCD imaging at all. We only used the quadratically summed DSS2 Red and Blue photographic plate data to overlay the ROSAT PSPC X-ray contours.

Cl J0254+0012 appears to be a collection of X-ray point sources. There is one galaxy at the edge of the field with an SDSS redshift $(z=0.35952)$ and the cluster of galaxies SDSS CE J043.601063+00.230312 has been detected at $z=0.32$ (estimated by the SDSS teams as indicated in NED) also at the edge of the field. We note that we used DSS2 data for C1 J0254+0012 and not SDSS data because this source is only located about 1 arcmin south of an SDSS covered area.

Cl J0302-1526 appears to be a collection of X-ray point sources with one apparent optical identification and is probably not a cluster of galaxies.

Cl J0317-0259 has an X-ray emission that is PSF dominated with one visible optical object within the X-ray contours. This source has also been detected in the ongoing XCS survey (Romer et al.: private communication and 2001) as an unextended source. This source is therefore possibly a real point

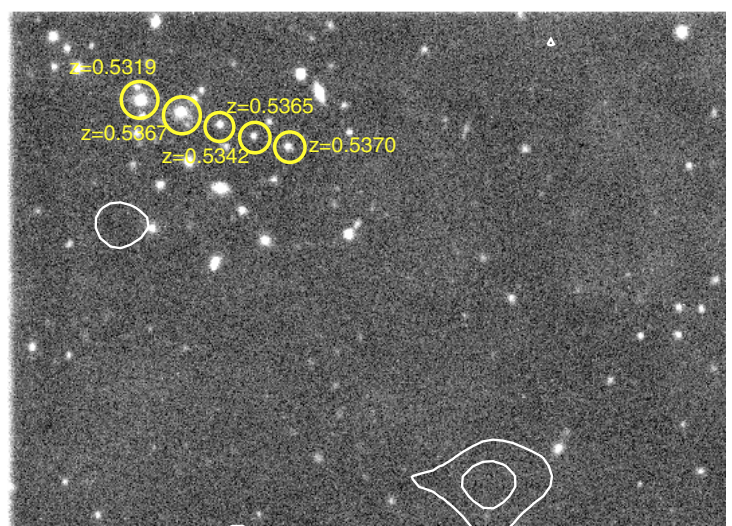

Fig. 9. A serendipitously detected galaxy structure in the Cl J1050+6317 field of view. White contours are X-ray ROSAT PSPC data.

source (given the better XMM angular resolution compared to the ROSAT PSPC) but its nature remains undeterminate.

\subsection{Additional candidates}

We found by chance another cluster of galaxies in the Cl J1050+6317 field of view (Fig. 9). This structure is clearly visible in the optical but completely invisible in the ROSAT PSPC data, implying that it is under luminous in X-rays. We measured five redshifts with the MOSCA instrument at the Calar Alto $3.5 \mathrm{~m}$ telescope. All proved to be around $z=0.535$ and they were mainly characterized by absorption lines (only one clearly shows the [OII] emission line). Five redshifts are not enough to give a robust velocity dispersion, but the raw computation gives a value of $430 \mathrm{~km} \mathrm{~s}^{-1}$. This structure is probably a moderately massive cluster. The brightest galaxy has an $i^{\prime}$ magnitude of 20.1, corresponding to an absolute magnitude of -22.3 which is typical for the central galaxy of a relatively rich galaxy structure.

We also probably found another galaxy structure east of the Cl J1113+4042 ROSAT X-ray source. This X-ray structure has not been sampled with optical spectroscopy but clearly appears associated with a galaxy concentration of a dozen galaxies. The brightest galaxy of this X-ray source has an $i^{\prime}$ magnitude of 19.3 (yielding a redshift in the [0.15-0.6] redshift range, depending on whether it is a group or a rich cluster).

\section{Discussion and conclusions}

We have given an update on our extended SHARC survey. Due to the large number of sources and significant amount of observing time needed to obtain redshifts and new X-ray measurements of all the objects, we have presented this work as an intermediate report, so as to make the data available to the public. For simplicity we will assume in our discussion that all the galaxy concentrations we have found are rich clusters, but we acknowledge that some of these could be groups or concentrations on the line of sight of active galaxies and are not necessarily gravitationally bound systems.

There are several interesting aspects to this work: (a) optical cluster searches versus X-ray observations, (b) how we compare with the recent ChaMP results (Barkhouse et al. 2006, also a work in progress); (c) how this relates to future missions designed to find clusters and/or AGN; and (d) the QSO population we found. 
Both Donahue et al. (2006) and Barkhouse et al. (2006) demonstrated that it is possible to find optical or near IR concentrations of galaxies that are probably clusters of galaxies, but that these can be weak X-ray emitters (see also Stanford et al. 2005). In our work, we have found the same, in that simply taking relatively deep (i.e. with exposure times $\sim 90 \mathrm{~min}$ in the $i^{\prime}$-band with 4 -m class telescopes) $3^{\prime} \times 3^{\prime}$ images can reveal clusters of galaxies in the $z=0.5-0.7$ range (see e.g. the serendipitously discovered cluster in the field of $\mathrm{Cl} \mathrm{J} 1050+6317)$. Our $i^{\prime}$ images were also large enough to encompass an area well outside the $\mathrm{X}$-ray image location, and we uncovered faint X-ray clusters and point sources in this process.

As shown by Brodwin et al. (2006), by moving further into the IR even more distant clusters can be found and photometric redshifts can be estimated. These low X-ray luminosity clusters may pose a potential puzzle: if they are massive, then their baryon fraction must be small compared to low redshift clusters. If this is the case, why is the hot gas missing? Hence, a possible quandary arises for those who want to use either X-ray or S-Z surveys to determine cluster evolution and for those using clusters as cosmological probes. For if these clusters have indeed significant amounts of matter, then these X-ray and S-Z invisible clusters must be taken into account when comparing predictions of cluster evolution with cosmological models. Thus picking out a set of these under-luminous $z \sim 0.5-0.6$ clusters from currently available data bases and measuring their velocity dispersions and/or gravitational lensing signal to determine masses will be very important when using cluster surveys to determine cosmological model parameters. This proposed project would create a census of the X-ray under luminous clusters to determine how their numbers compare to those of X-ray luminous clusters.

Besides the optical and near IR observations, X-ray observations have been one of the standard methods used for finding clusters of galaxies. The examination of the field outside the pointing center of X-ray observations has also been used for a long time (e.g. Henry et al. 1992). There are too many references to review and compare with all the results of these works. We therefore confine ourselves to comparing our work with a very recent survey by Barkhouse et al. (2006), who surveyed $13 \mathrm{deg}^{2}$ down to a flux limit of about $1.5 \times 10^{-14} \mathrm{erg} \mathrm{cm}^{-2} \mathrm{~s}^{-1}$. They found $\sim 2.5 \mathrm{X}$-ray cluster candidates per $\mathrm{deg}^{2}$ with no optical counterpart. We estimate the areal coverage of our current survey to be $\sim 15 \mathrm{deg}^{2}$ from Fig. A.12 of Adami et al. (2000) at the sensitivity level of about $2.3 \times 10^{-14} \mathrm{erg} \mathrm{cm}^{-2} \mathrm{~s}^{-1}$. Thus, we have found comparable numbers of $\mathrm{X}$-ray cluster candidates compared to the Barkhouse et al. survey: 1.8 per $\mathrm{deg}^{2}$. This value is based on a flux limit of $2.3 \times 10^{-14} \mathrm{erg} \mathrm{cm}^{-2} \mathrm{~s}^{-1}$ (compared to the $1.5 \times 10^{-14} \mathrm{erg} \mathrm{cm}^{-2} \mathrm{~s}^{-1}$ of Barkhouse et al. 2006), and on all the confirmed clusters plus the candidates with a pending status in Table 1. Furthermore, in Fig. 10, it can also be seen that the redshift distribution of the clusters and cluster candidates of our work and of Barkhouse et al. are similar.

This naturally leads to the question for future surveys as to what is the best approach in terms of overall design for an X-ray telescope. For example, the proposed VADER mission concept (Fassbender et al. 2006) uses the flight spare XMM-Newton mirrors that would allow an expanded and curved detector array to cover an extended field of about 1 sq. degree. Although the XMM-Newton detectors cover a $1 \mathrm{deg}^{2}$ FOV, the vignetting caused by the outer mirror graze angle (inner mirrors have even smaller graze angles) of about 30 arcmin results in a region of about $20 \times 20$ arcmin $^{2}$ where the effective area is $\gtrsim 50 \%$. In contrast, a telescope that only works below about $2 \mathrm{keV}$ such as

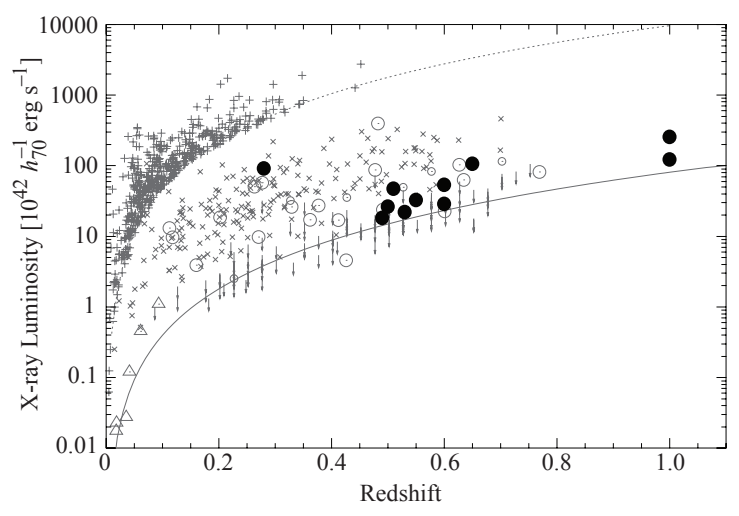

Fig. 10. Figure 10 of Barkhouse et al. (2006) showing the distribution of X-ray luminosity $(0.5-2.0 \mathrm{keV})$ as a function of redshift. Open circles are the Barkhouse et al. (2006) extended X-ray sources associated with clusters and the open triangles are extended X-ray sources associated with nearby galaxies. Other symbols are from literature studies (see Barkhouse et al. 2006, for details). Our data are shown with large black filled circles.

ROSAT, but with an expanded area made possible by using thin $(\sim 0.5 \mathrm{~mm})$ electro formed mirrors, for example, would be able to cover nearly $1 \times 1 \mathrm{deg}^{2}$ with minimal vignetting and a focal length about 2-2.5 times shorter (important both for cost and for reducing the detector background for extended sources); with a design to optimize the off axis angular resolution, the survey would be approximately 10 times the coverage as the VADER design (e.g. Harvey et al. 2004; Atanassova \& Harvey 2003; Citterio et al. 1999; Ulmer 1995; and Burrows et al. 1992).

It is the distant clusters $(z \gtrsim 1)$ that are most important to find, and their apparent temperature, $T_{\text {apparent }}=T_{\text {intrinsic }} /(1+z)$ will be approximately $3 \mathrm{keV}$ (or less), corresponding to intrinsic temperatures of $6 \mathrm{keV}$ (or less). Hence most of these distant clusters can be found without using X-ray telescopes that have significant effective area above about $6 \mathrm{keV}$. We have also shown that even with reduced off axis angular resolution, clusters can be found. The major drawback to a mission with new mirrors is having to design in detail and then fabricate new mandrels and mirrors. In comparison, for the VADER mission, the X-ray telescopes already exist, and the full array of IR to Optical to $\mathrm{X}$-ray telescopes combine to make the proposed capabilities of the VADER mission concept impressive.

AGN/QSOs surveys would also benefit from an X-ray survey mission even if it is one that only works below about $2 \mathrm{keV}$. Although AGN/QSOs may be heavily absorbed (e.g. have spectra that decrease with decreasing energy due to photoelectric absorption in the rest frame at about $4 \mathrm{keV}$ ), QSOs (at least optically identified ones) show a peak in their redshift distribution at about $z=2$ (e.g. Schneider et al. 2005). The $4 \mathrm{keV}$ absorption will be moved down to $1.3 \mathrm{keV}$ for these QSOs. Also, many of those at even lower redshifts should be easily found with a $0.5-2 \mathrm{keV}$ survey.

In conclusion, a combination of deep $i^{\prime}$ band images and $\mathrm{X}$-ray images is a productive way to find more clusters. Some of the $i^{\prime}$-band images (or a deep $i^{\prime}$ survey) will likely produce $X$-ray faint (and under luminous) clusters that are not coincident with the X-ray image. The under luminous X-ray clusters could give us new understanding of both the formation of the Intra Cluster Medium (or the lack there of) and the number of massive systems which could be missed by using X-ray (or S-Z) surveys alone. Furthermore, future missions that are aimed at searching for both distant rich clusters and QSOs in X-rays should 
seriously consider a wide field of view $\left(1 \times 1 \mathrm{deg}^{2}\right.$ or more) telescope design optimized for the $\sim 0.5-2 \mathrm{keV}$ range.

Acknowledgements. The authors thank the referee for his/her comments. We thank J.C. Cuillandre for providing us with the CFHT/CH12K data for Cl J1120+1254 and M.A. Hosmer for comparisons with the XCS-DR1 database. We also thank Calar Alto Observatory for allocation of director's discretionary time to this programme. This paper is based on observations: 1) Obtained with the Apache Point Observatory $3.5 \mathrm{~m}$ telescope, owned and operated by the Astrophysical Research Consortium. 2) Obtained at the Canada-France-Hawaii Telescope (CFHT) operated by the National Research Council of Canada, the Institut National des Sciences de l'Univers of the Centre National de la Recherche Scientifique of France, and the University of Hawaii. 3) Obtained at the Gemini Observatory, which is operated by the Association of Universities for Research in Astronomy, Inc., under a cooperative agreement with the NSF on behalf of the Gemini partnership: the National Science Foundation (United States), the Particle Physics and Astronomy Research Council (UK), the National Research Council (Canada), CONICYT (Chile), the Australian Research Council (Australia), CNPq (Brazil) and CONICET (Argentina). 4) Observations collected at the German-Spanish Astronomical Center, Calar Alto, jointly operated by the Max-Planck-Institut für Astronomie Heidelberg and the Instituto de Astrofísica de Andalucía (CSIC). 5) Observations made with ESO Telescopes at the La Silla and Paranal Observatories. 6) SDSS data: Funding for the SDSS and SDSS-II has been provided by the Alfred P. Sloan Foundation, the Participating Institutions, the National Science Foundation, the US Department of Energy, the National Aeronautics and Space Administration, the Japanese Monbukagakusho, the Max Planck Society, and the Higher Education Funding Council for England. The SDSS Web Site is http://www.sdss.org/. The SDSS is managed by the Astrophysical Research Consortium for the Participating Institutions. The Participating Institutions are the American Museum of Natural History, Astrophysical Institute Potsdam, University of Basel, University of Cambridge, Case Western Reserve University, University of Chicago, Drexel University, Fermilab, the Institute for Advanced Study, the Japan Participation Group, Johns Hopkins University, the Joint Institute for Nuclear Astrophysics, the Kavli Institute for Particle Astrophysics and Cosmology, the Korean Scientist Group, the Chinese Academy of Sciences (LAMOST), Los Alamos National Laboratory, the Max-Planck-Institute for Astronomy (MPIA), the Max-Planck-Institute for Astrophysics (MPA), New Mexico State University, Ohio State University, University of Pittsburgh, University of Portsmouth, Princeton University, the United States Naval Observatory, and the University of Washington. Also based on observations made at Observatoire de Haute Provence (CNRS), France.

This research has made use of the SIMBAD database, operated at CDS, Strasbourg, France, and of the NASA/IPAC Extragalactic Database (NED) which is operated by the Jet Propulsion Laboratory, California Institute of Technology, under contract with the National Aeronautics and Space Administration.

\section{References}

Adami, C., Ulmer, M. P., Romer, A. K., et al. 2000, ApJS, 131, 391

Adami, C., Picat, J. P., Savine, C., et al. 2006a, A\&A, 451, 1159

Adami, C., Scheidegger, R., Ulmer, M. P., et al. 2006b, A\&A, 459, 679

Allen, S., Schmidt, R. W., Ebeling, H., Fabian, A. C., \& van Speybroeck, L. 2004, MNRAS, 353, 457

Andreon, S., Willis, J., Quintana, H., et al. 2004, MNRAS, 353, 353

Atanssova, M., \& Harvey, J. E. 2003, SPIE, 5226, 275

Barkhouse, W. A., Green, P. J., Vikhlinin, A., et al. 2006, ApJ, 645, 955

Burke, D., Collins, C., Sharples, R., et al. 1997, ApJ, 488, L83

Burrows, C. J., Burg, R., \& Giacconi, R. 1992, ApJ, 392, 760

Brodwin, M., Brown, M. J. I., Ashby, M. L. N., et al. 2006, ApJ, 651, 791

Citterio, O., Campana, S., Conconi, P., et al. 1999, SPIE, 3766, 198

Dickey, J. M., \& Lockman, F. J. 1990, ARA\&A, 28, 215

Donahue, M., Horner, D. J., Cavagnolo, K. W., \& Voit, G. M. 2006, ApJ, 643, 730

Ebeling, H., Edge, A., Bohringer, H., et al. 1998, MNRAS, 301, 881

Fassbender, R., Stegmaier, J., Weijmans, A.-M., et al. 2006, SPIE, 6266, 90

Fukugita, M., Shimasaku, K., \& Ichikawa, T. 1995, PASP, 107, 945

Harvey, J. E., Atanssova, M., \& Krywonos, A. 2004, SPIE, 5497, 636

Henry, J. P., Gioia, I. M., Maccacaro, T., et al. 1992, ApJ, 386, 408

Henry, J. P., Gioia, I. M., Mullis, C., et al. 1997, AJ, 114, 1293

Holden, B. P., Stanford, S. A., Eisenhard, P., \& Dickinson, M. 2004, AJ, 127, 2484

Jones, L. R., Ponman, T. J., Horton, A., et al. 2003, MNRAS, 343, 627

Lasker, B. M., Sturch, C. R., McLean, B. J., et al. 1990, AJ, 99, 2019

Le Fèvre, O., Saisse, M., Mancini, D., et al. 2003, SPIE, 4841, 1670

McLean, B. J., Greene, G. R., Lattanzi, M. G., \& Pirenne, B. 2000, ASPC, 216, 145

Nichol, R. C., Romer, A. K., Holden, B. P., et al. 1999, ApJ, 521, L21

Penprase, B. E., Rhodes, J. D., \& Harris, E. L. 2000, A\&A, 364, 712

Pierre, M., Pacaud, F., \& Duc, P. A. 2006, MNRAS, 372, 591

Romer, A., Nichol, R., Holden, B., et al. 2000, ApJS, 126, 209

Romer, A., Viana, P., Liddle, A., \& Mann, R. 2001, ApJ, 547, 594

Rosati, P., Della Ceca, R., Norman, C., et al. 1998, ApJ, 492, 21

Sarazin, C. 1986, Rev. Mod. Phys., 58, 1

Scharf, C., Jones, L., Ebeling, H., et al. 1997, ApJ, 477, 79

Schneider, D. P., Hall, P. B., Richards, G. T., et al. 2005, 130, 367

Stanford, S. A., Eisenhardt, P. R., Brodwin, M., et al. 2005, ApJ, 634, L129

Szalay, A. S., Connolly, A. J., \& Szokoly, G. P. 1999, AJ, 117, 68

Ulmer, M. P. 1995, SPIE, 2515, 280

Ulmer, M. P., Adami, C., Covone, G., et al. 2005, ApJ, 624, 124

Vikhlinin, A., McNamara, B., Forman, W., et al. 1998, ApJ, 502, 558

White, R. L., Becker, R. H.., Helfand, D. J., \& Gregg, M. D. 1997, ApJ, 475, 479 
C. Adami et al.: An extended SHARC survey, Online Material $p 1$

\section{Online Material}


C. Adami et al.: An extended SHARC survey, Online Material p 2

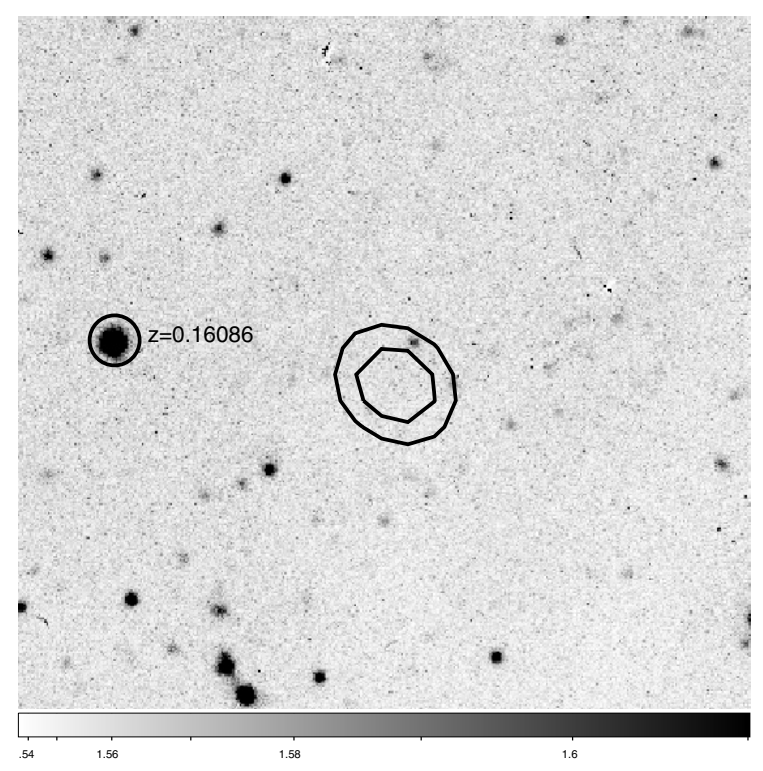

Fig. A.1. $R$ band OHP image for Cl J0223-0856 (completeness level: $R \sim 20$ ). The field is $4.2 \times 4.2 \mathrm{arcmin}^{2}$.

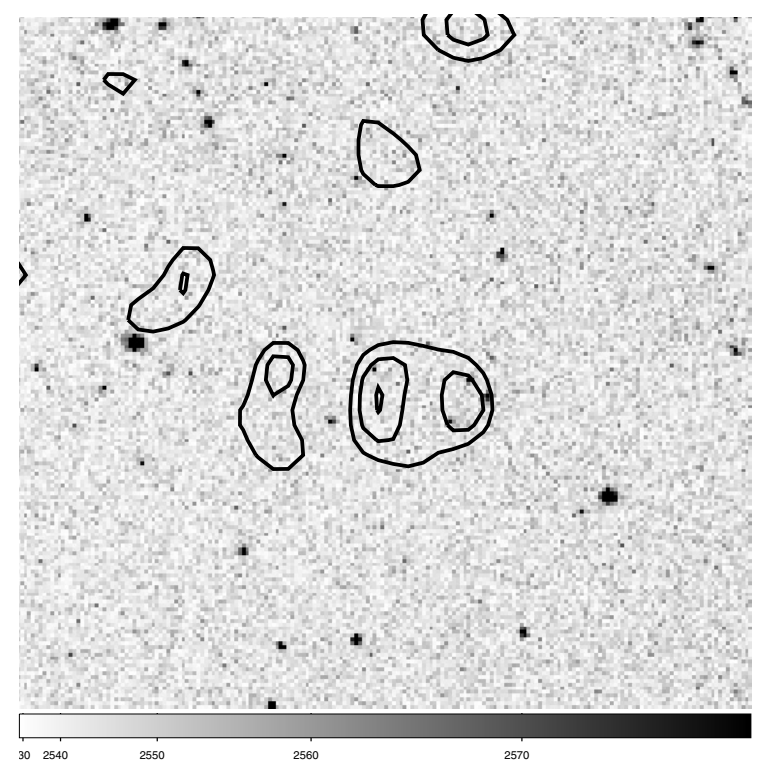

Fig. A.2. $\chi^{2}$ image for Cl J0240-0801 built from the SDSS $u, g^{\prime}, r^{\prime}, i^{\prime}$ and $z^{\prime}$ images. The field is $3.7 \times 3.7 \mathrm{arcmin}^{2}$.

\section{Appendix A}

We present in this appendix the 36 optical images of our candidates overlayed with X-ray contours (ROSAT data except when quoted). We also give the known redshifts in the given optical area.

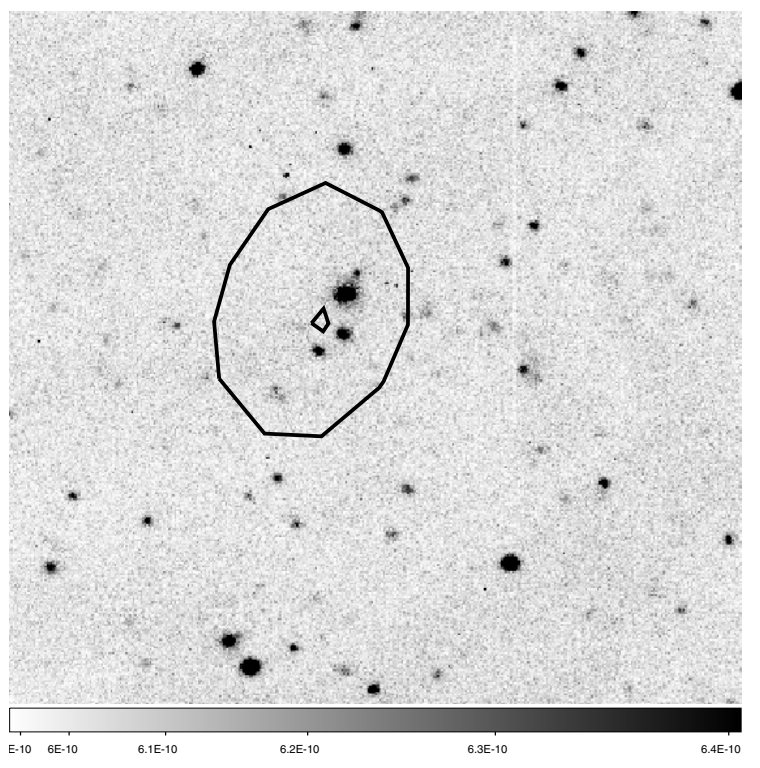

Fig. A.3. $I$ image for Cl J0241-0802 observed at ESO (completeness level: $I \sim 21)$. The field is $2 \times 2 \operatorname{arcmin}^{2}$.

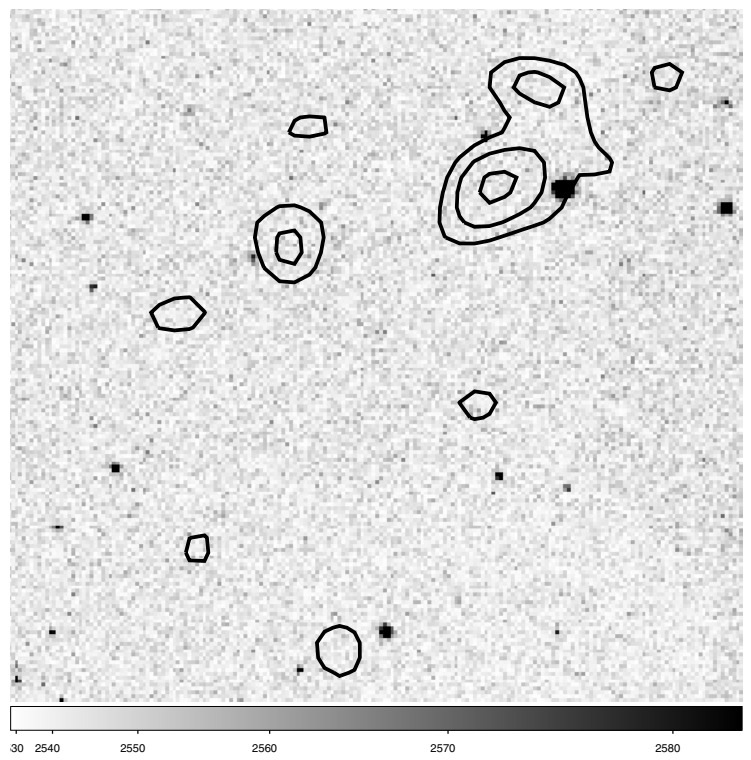

Fig. A.4. $\chi^{2}$ image for Cl J0242-0756 built from the SDSS $u, g^{\prime}, r^{\prime}, i^{\prime}$ and $z^{\prime}$ images. The field is $3.7 \times 3.7 \mathrm{arcmin}^{2}$. 
C. Adami et al.: An extended SHARC survey, Online Material p 3

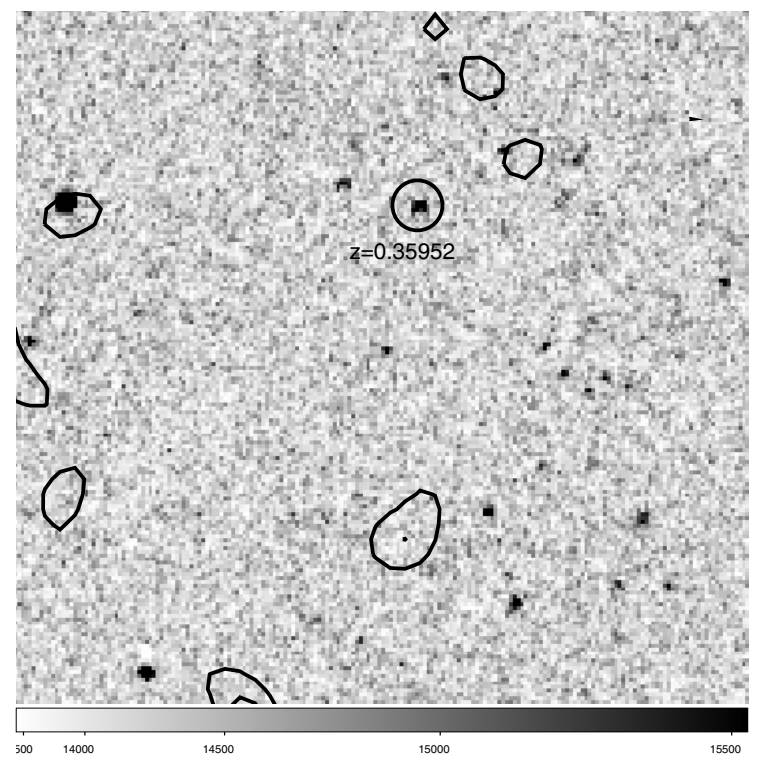

Fig. A.5. $\chi^{2}$ image for $\mathrm{Cl} \mathrm{J} 0254+0012$ built from the DSS red and blue images. The field is $3.7 \times 3.7 \mathrm{arcmin}^{2}$.

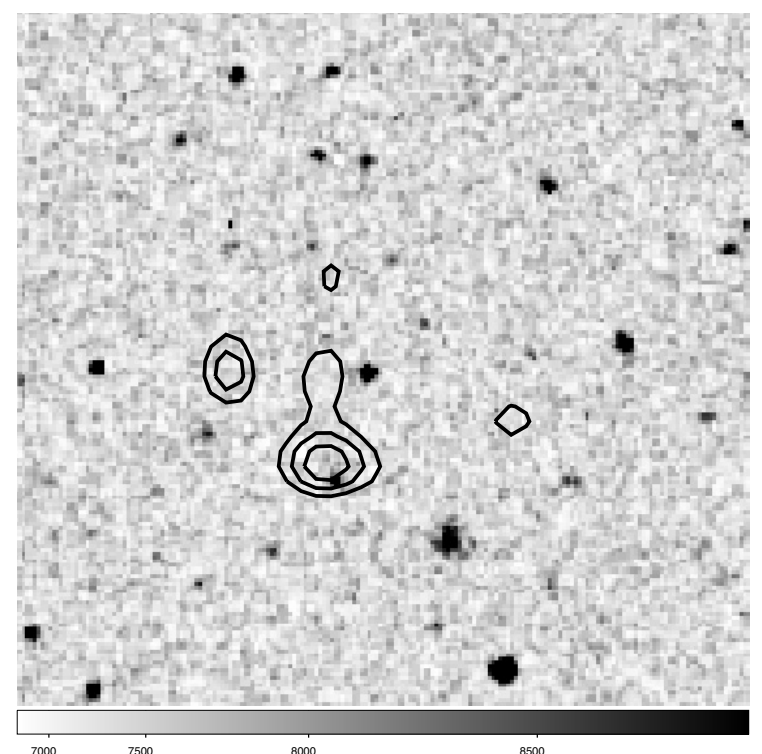

Fig. A.6. $\chi^{2}$ image for Cl J0302-1526 built from the DSS red and blue images. The field is $3.7 \times 3.7 \mathrm{arcmin}^{2}$.

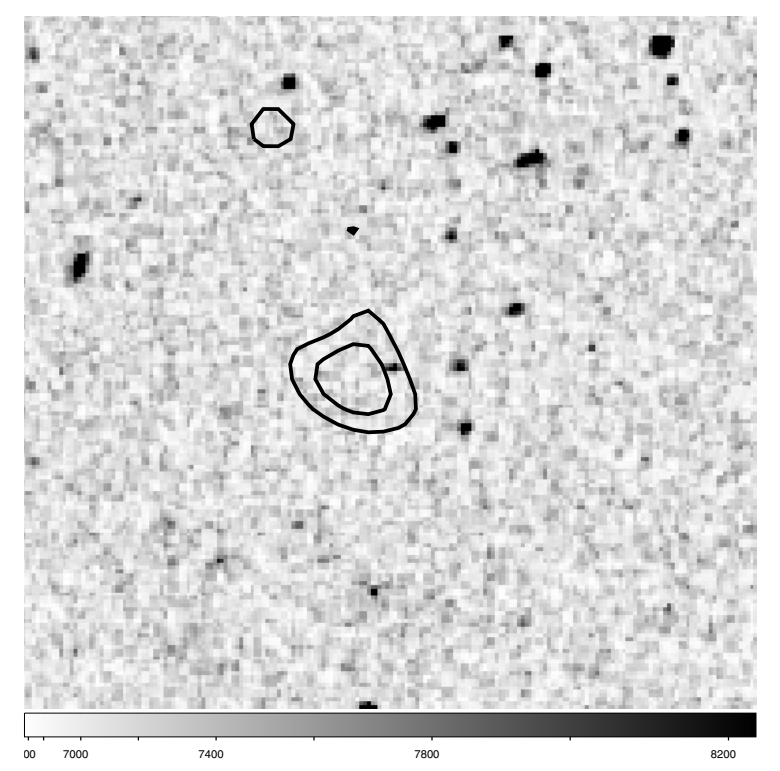

Fig. A.7. $\chi^{2}$ image for Cl J0317-0259 built from the DSS red and blue images. The field is $3.7 \times 3.7 \mathrm{arcmin}^{2}$.

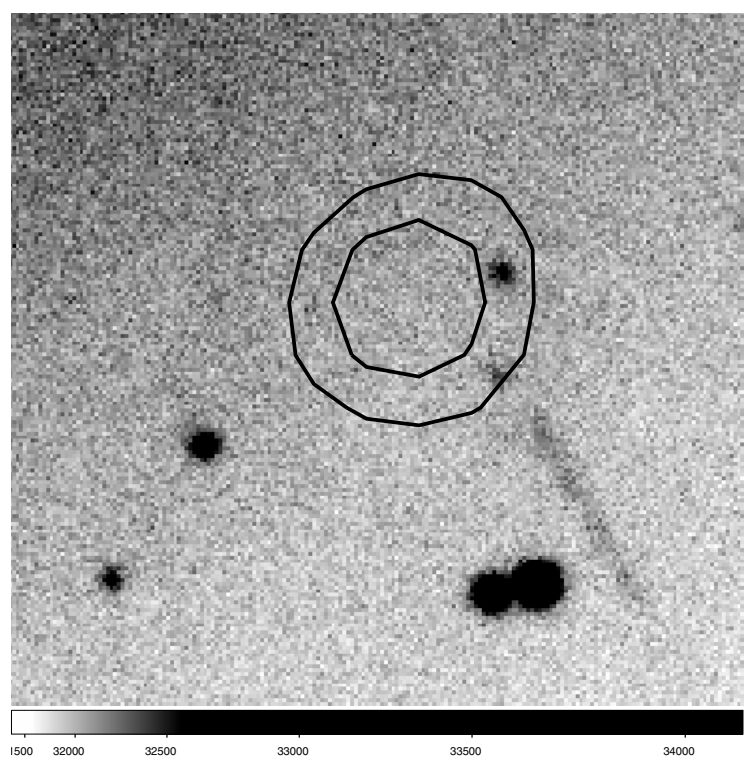

Fig. A.8. $R$ band OHP image for $\mathrm{Cl} \mathrm{J} 0413+1215$ (completeness level: $R \sim 21.5)$. The field is $4.2 \times 4.2 \operatorname{arcmin}^{2}$. 
C. Adami et al.: An extended SHARC survey, Online Material $p 4$

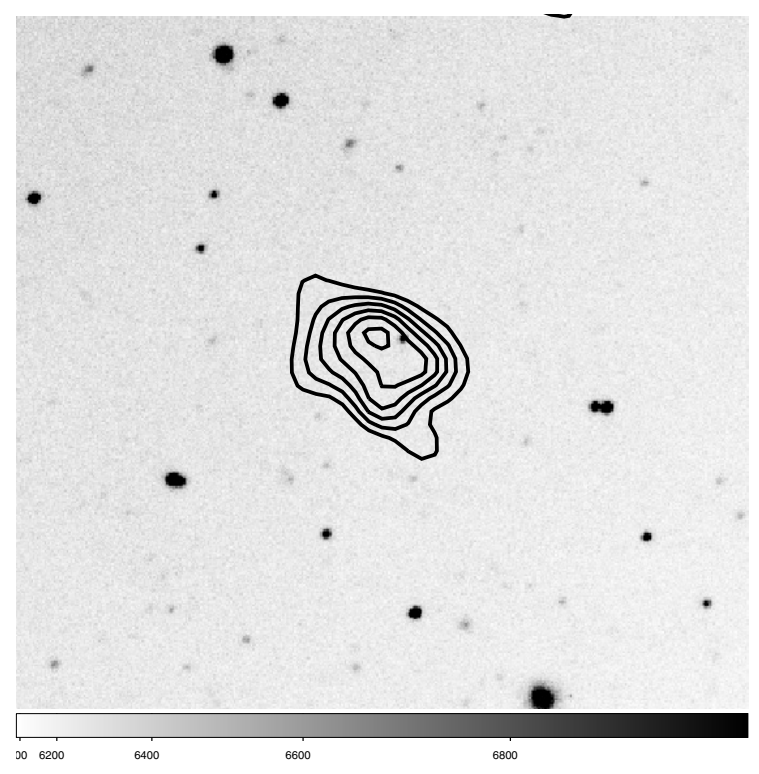

Fig. A.9. $R$ band OHP image for $\mathrm{Cl} \mathrm{J} 0922+6217$ (completeness level: $R \sim 20$ ). The field is $4.2 \times 4.2 \mathrm{arcmin}^{2}$.

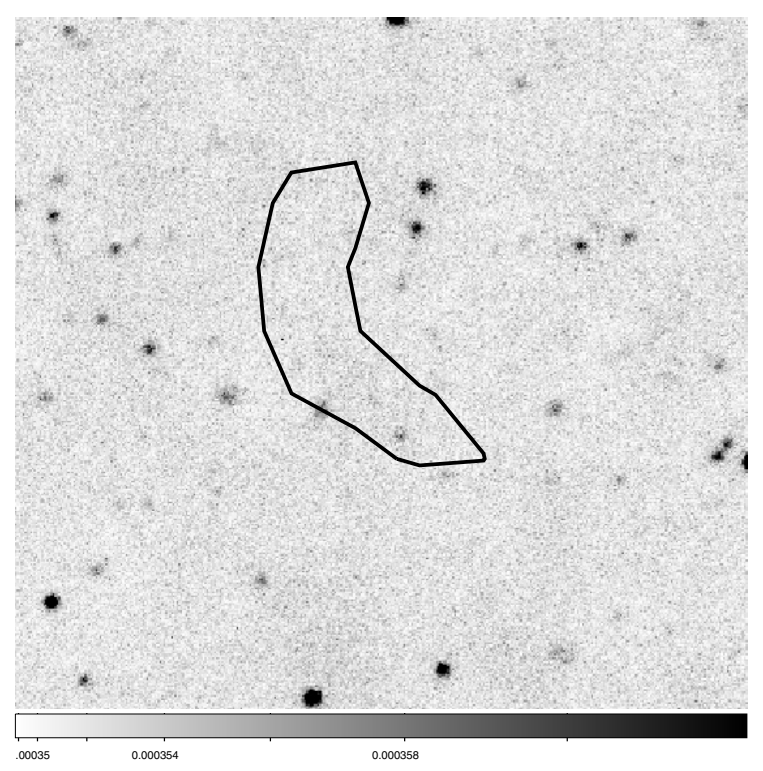

Fig. A.10. $i^{\prime}$ image for $\mathrm{Cl} \mathrm{J0937+6105} \mathrm{observed} \mathrm{at} \mathrm{ARC} \mathrm{(completeness}$ level: $i^{\prime} \sim 22.5$ ). The field is $1.8 \times 1.8 \mathrm{arcmin}^{2}$.

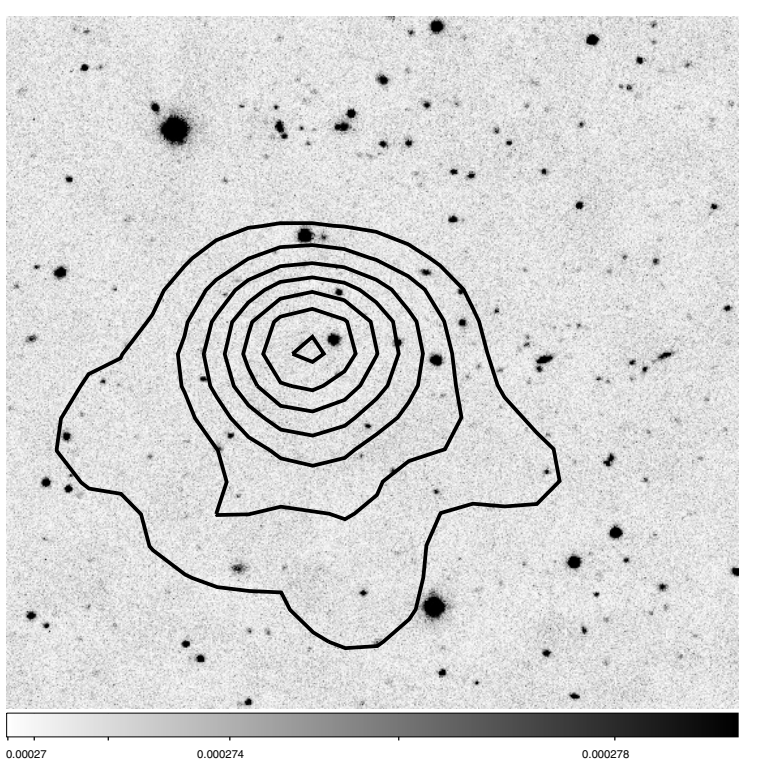

Fig. A.11. $i^{\prime}$ image for $\mathrm{Cl} \mathrm{J} 1024+1935$ observed at ARC (completeness level: $\left.i^{\prime} \sim 23\right)$. The field is $3 \times 3 \operatorname{arcmin}^{2}$.

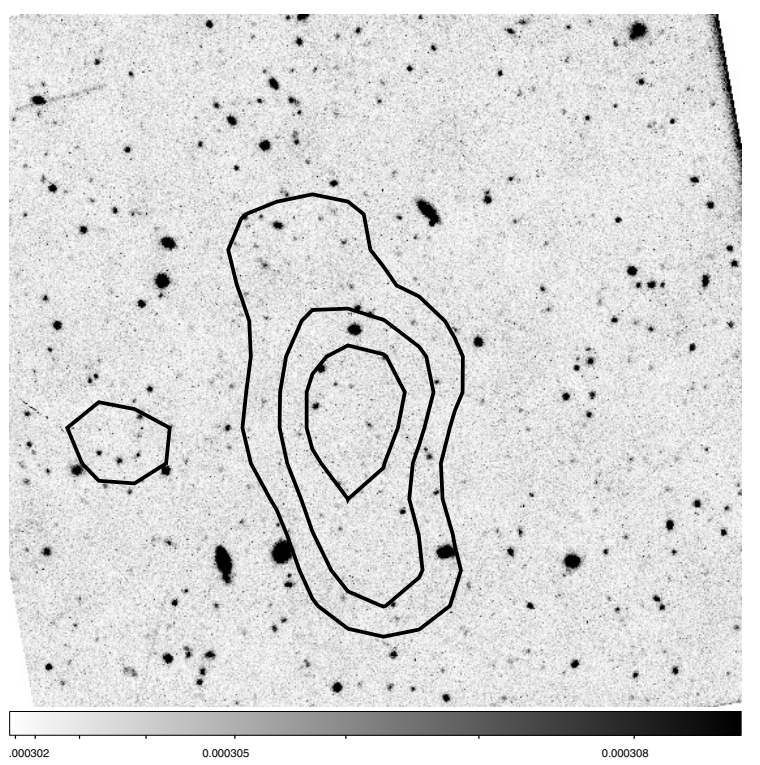

Fig. A.12. $i^{\prime}$ image for $\mathrm{Cl} \mathrm{J} 1024+1943$ observed at ARC (completeness level: $\left.i^{\prime} \sim 23.5\right)$. The field is $3 \times 3 \operatorname{arcmin}^{2}$. 
C. Adami et al.: An extended SHARC survey, Online Material p 5

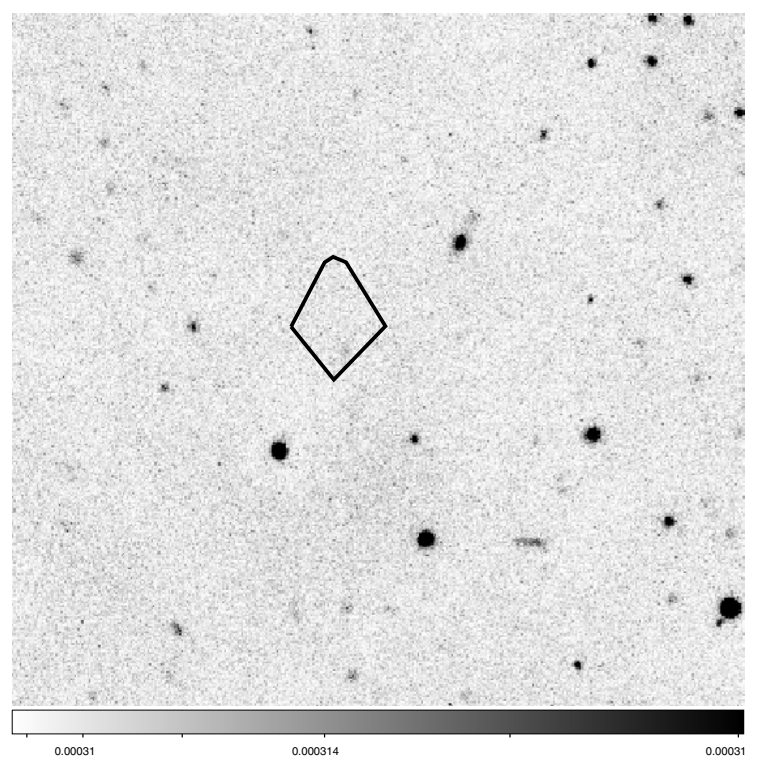

Fig. A.13. $i^{\prime}$ image for $\mathrm{Cl} \mathrm{J} 1050+6317$ observed at ARC (completeness level: $\left.i^{\prime} \sim 23\right)$. The field is $1.8 \times 1.8 \mathrm{arcmin}^{2}$.

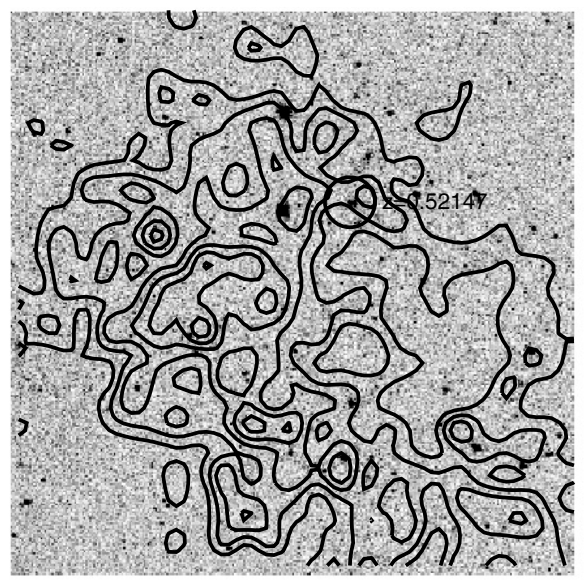

Fig. A.14. $\chi^{2}$ image for $\mathrm{Cl} \mathrm{J} 1052+5655$ built from the SDSS $u, g^{\prime}, r^{\prime}, i^{\prime}$ and $z^{\prime}$ images. The field is $5.9 \times 5.9 \mathrm{arcmin}^{2}$.

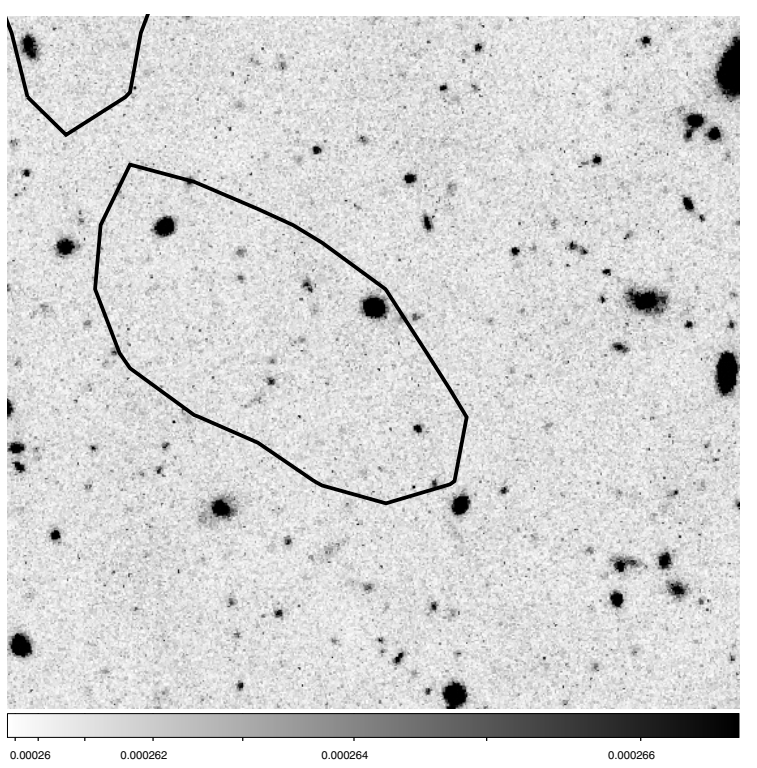

Fig. A.15. $i^{\prime}$ image for $\mathrm{Cl} \mathrm{J} 1052+5400$ observed at ARC (completeness level: $\left.i^{\prime} \sim 24\right)$. The field is $1.8 \times 1.8 \mathrm{arcmin}^{2}$. The X-ray source is from ROSAT data but is not confirmed by the available XMM data.

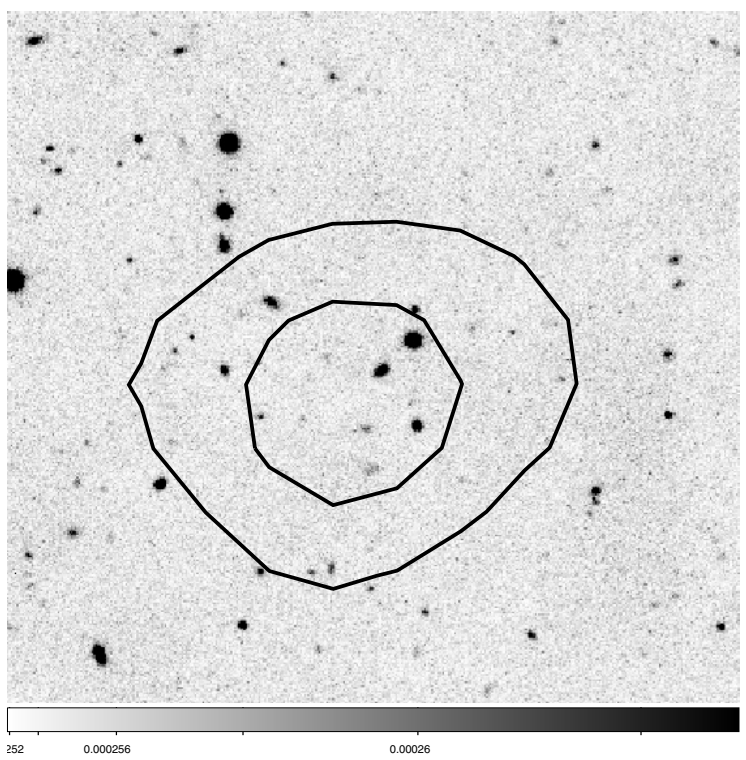

Fig. A.16. $i^{\prime}$ image for $\mathrm{Cl} \mathrm{J} 1102+2514$ observed at ARC (completeness level: $\left.i^{\prime} \sim 23\right)$. The field is $1.8 \times 1.8 \mathrm{arcmin}^{2}$. 
C. Adami et al.: An extended SHARC survey, Online Material p 6

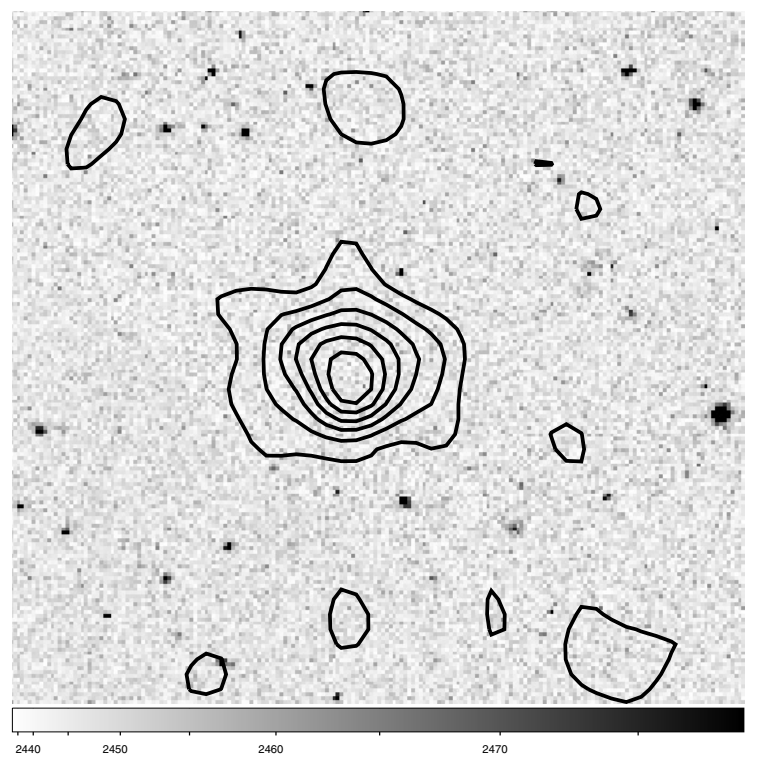

Fig. A.17. $\chi^{2}$ image for $\mathrm{Cl} \mathrm{J} 1103+2458$ built from the SDSS $u, g^{\prime}, r^{\prime}, i^{\prime}$ and $z^{\prime}$ images. The field is $3.7 \times 3.7 \mathrm{arcmin}^{2}$.

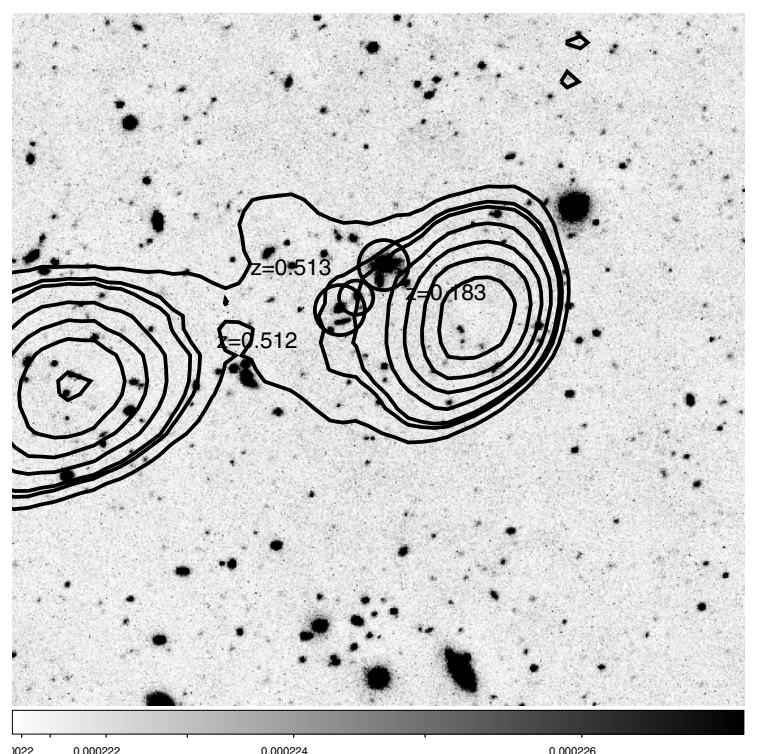

Fig. A.18. $i^{\prime}$ image for $\mathrm{Cl} \mathrm{J} 1113+4042$ observed at ARC (completeness level: $i^{\prime} \sim 23.5$ ). The field is $3 \times 3 \operatorname{arcmin}^{2}$. Overlayed X-ray contours are Chandra data.

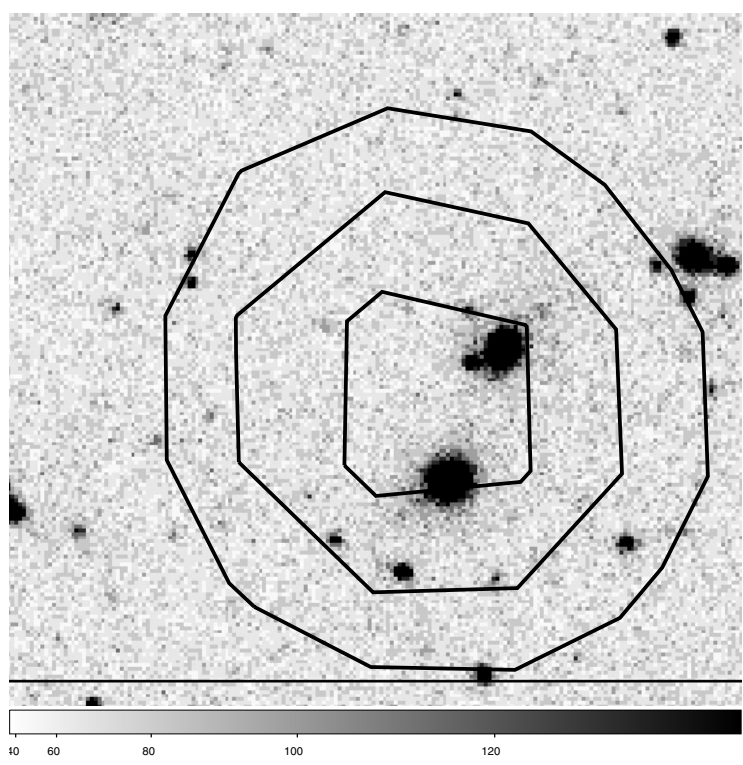

Fig. A.19. $R$ image for $\mathrm{Cl} \mathrm{J} 1120+1254$ observed at CFHT (completeness level: $R \sim 22$ ). The field is $1.8 \times 1.8 \operatorname{arcmin}^{2}$. Overlayed X-ray contours are XMM data.

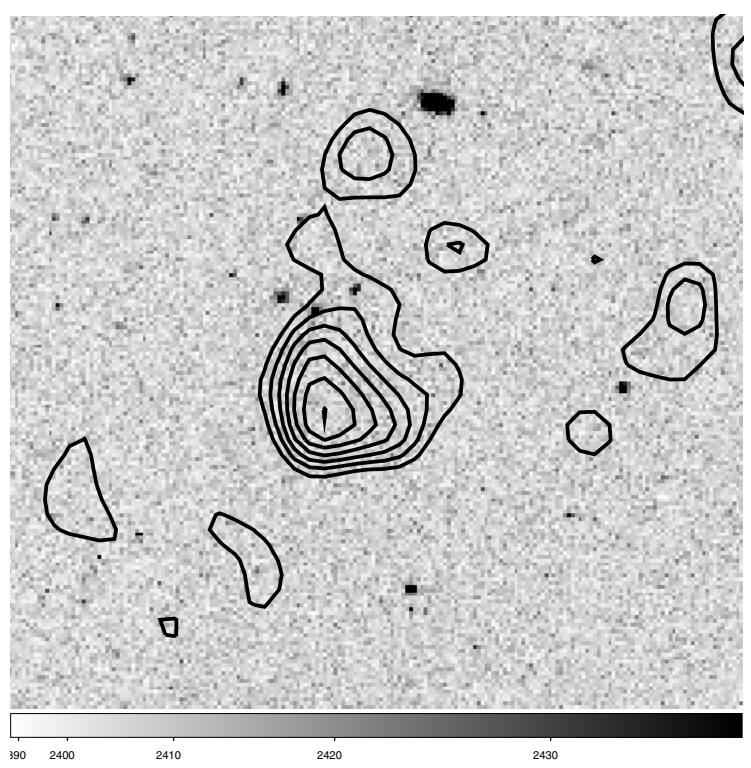

Fig. A.20. $\chi^{2}$ image for $\mathrm{Cl} \mathrm{J} 1121+4309$ built from the SDSS $u, g^{\prime}, r^{\prime}, i^{\prime}$ and $z^{\prime}$ images. The field is $3.7 \times 3.7 \mathrm{arcmin}^{2}$. 
C. Adami et al.: An extended SHARC survey, Online Material $p 7$

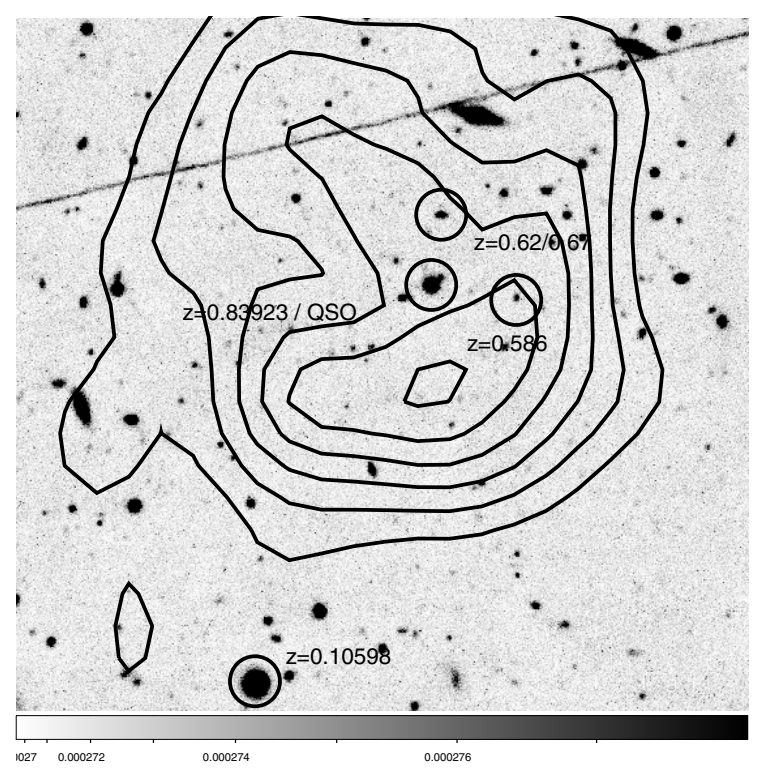

Fig. A.21. $i^{\prime}$ image for $\mathrm{Cl} \mathrm{J} 1121+0338$ observed at ARC (completeness level: $\left.i^{\prime} \sim 23.5\right)$. The field is $3 \times 3 \operatorname{arcmin}^{2}$.

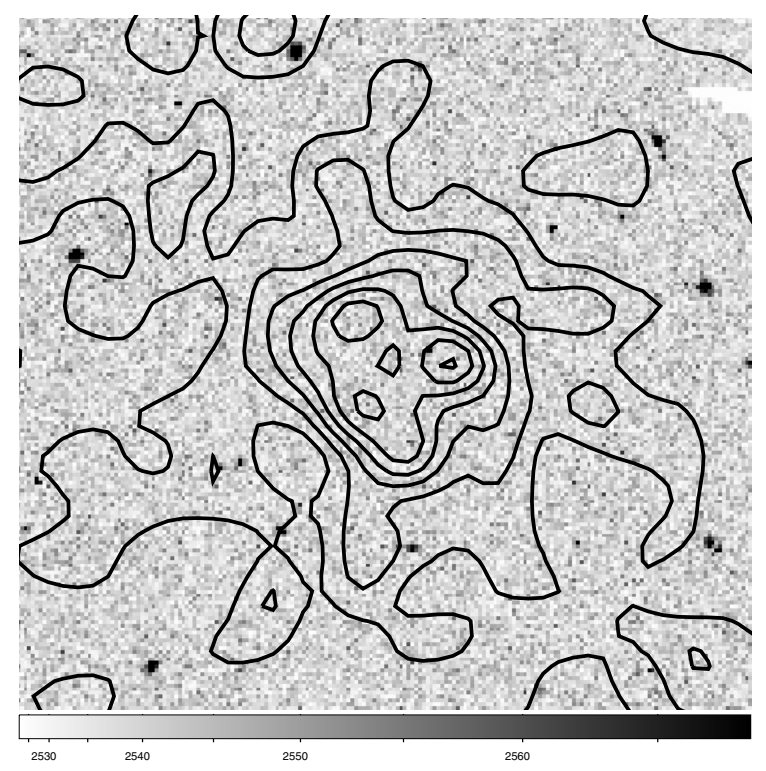

Fig. A.22. $\chi^{2}$ image for Cl J1158+5541 built from the SDSS $u, g^{\prime}, r^{\prime}, i^{\prime}$ and $z^{\prime}$ images. The field is $3.7 \times 3.7 \mathrm{arcmin}^{2}$. Overlayed X-ray contours are XMM data.

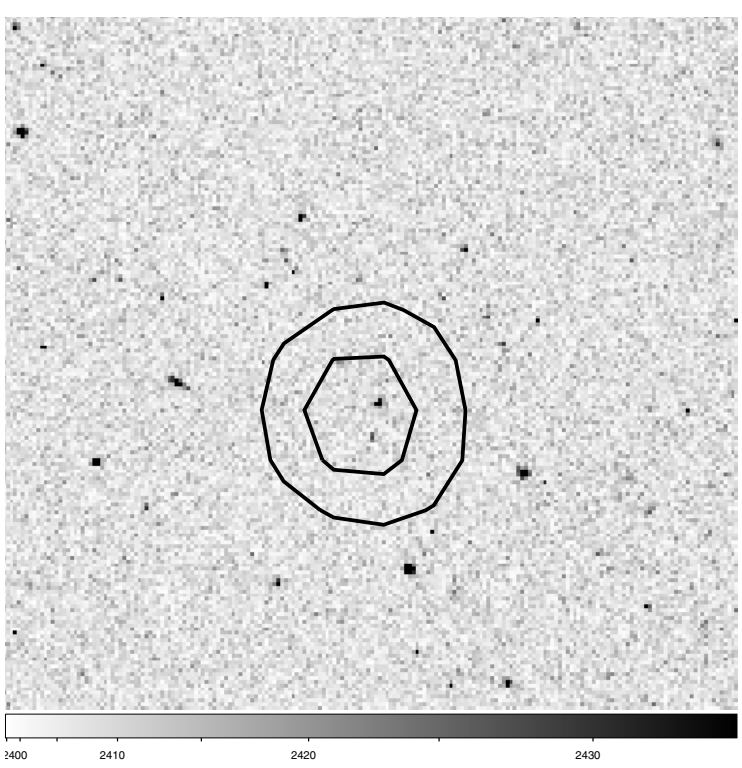

Fig. A.23. $\chi^{2}$ image for $\mathrm{Cl} \mathrm{J} 1202+4439$ built from the SDSS $u, g^{\prime}, r^{\prime}, i^{\prime}$ and $z^{\prime}$ images. The field is $3.7 \times 3.7 \operatorname{arcmin}^{2}$. Overlayed X-ray contours are XMM data.

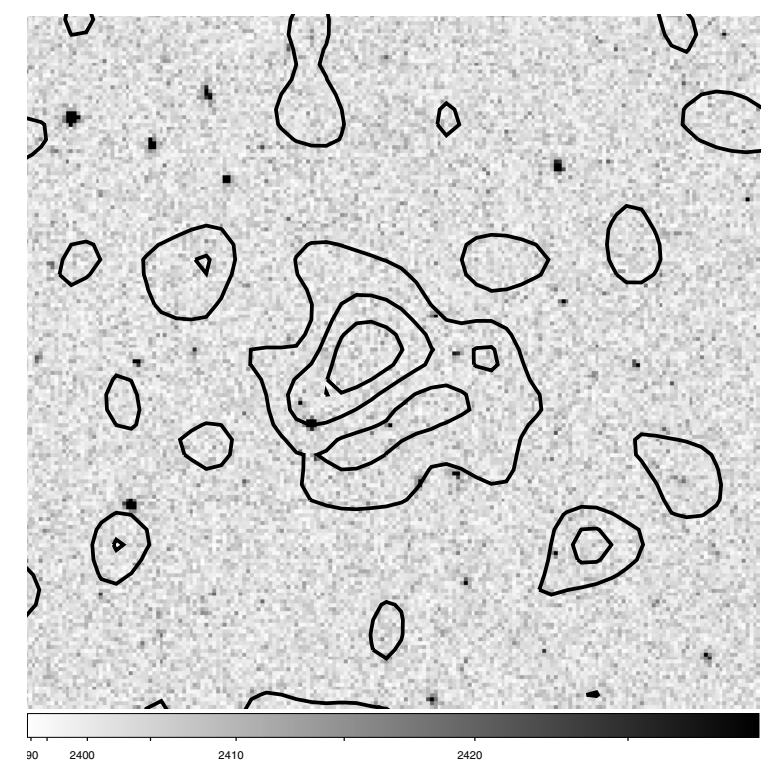

Fig. A.24. $\chi^{2}$ image for $\mathrm{Cl} \mathrm{J} 1207+4429$ built from the SDSS $u, g^{\prime}, r^{\prime}, i^{\prime}$ and $z^{\prime}$ images. The field is $3.7 \times 3.7 \mathrm{arcmin}^{2}$. 
C. Adami et al.: An extended SHARC survey, Online Material $p 8$

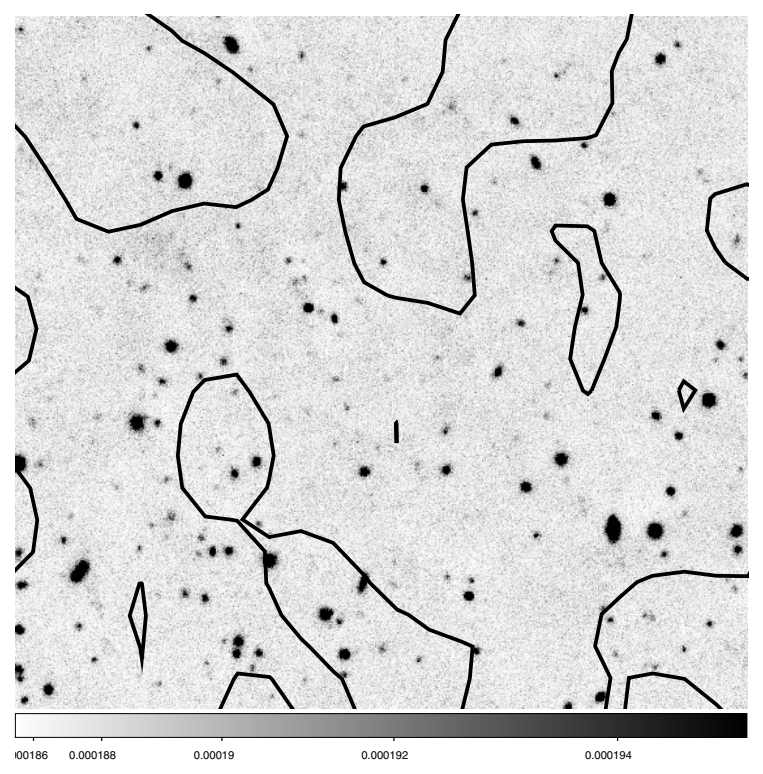

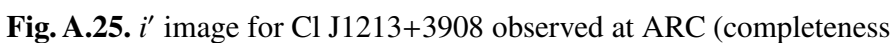
level: $i^{\prime} \sim 22.5$ ). The field is $3 \times 3 \operatorname{arcmin}^{2}$.

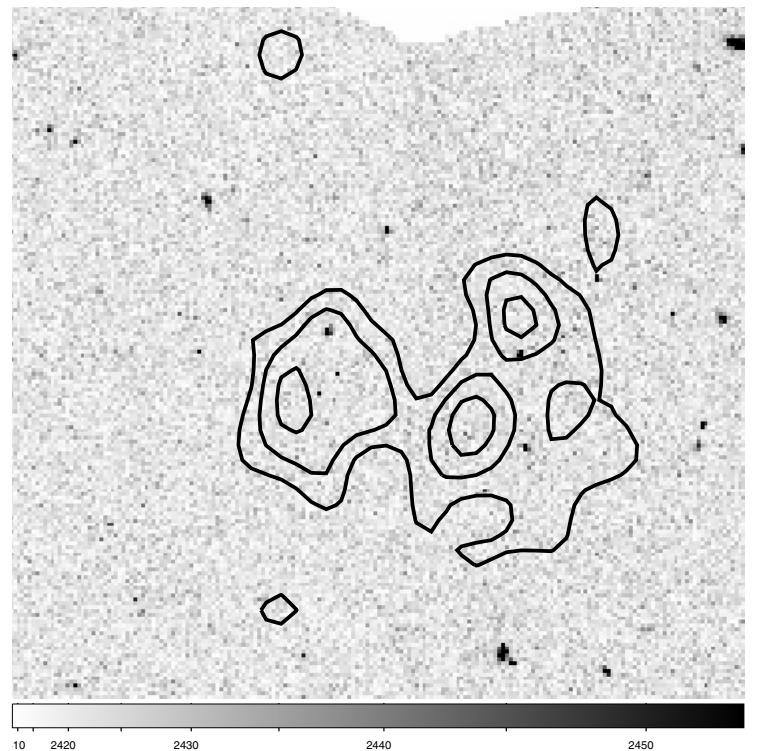

Fig. A.26. $\chi^{2}$ image for $\mathrm{Cl} \mathrm{J} 1213+3317$ built from the SDSS $u, g^{\prime}, r^{\prime}, i^{\prime}$ and $z^{\prime}$ images. The field is $3.7 \times 3.7 \operatorname{arcmin}^{2}$.

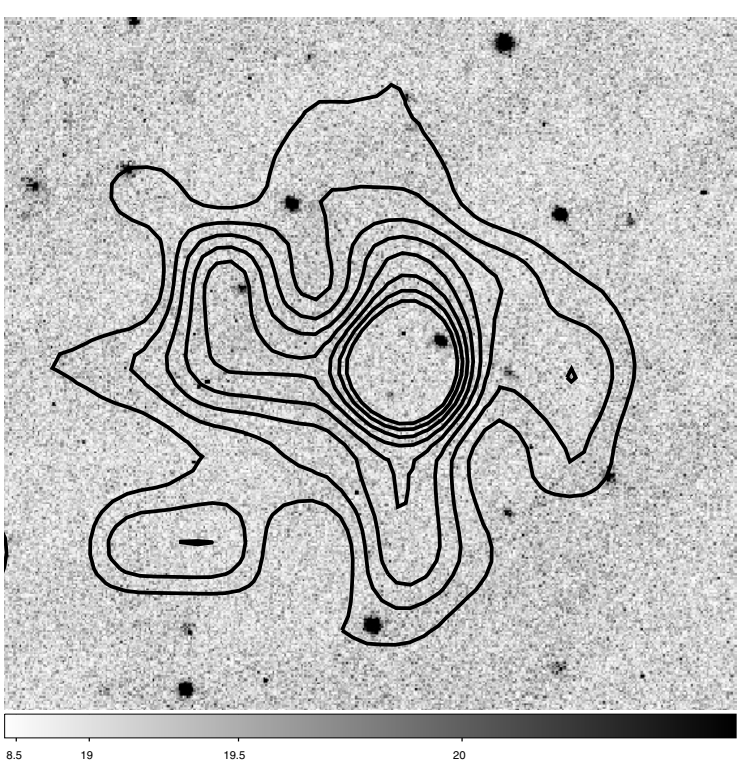

Fig. A.27. I image for Cl J1214+1254 observed at ESO (completeness level: $I \sim 21)$. The field is $2 \times 2 \operatorname{arcmin}^{2}$.

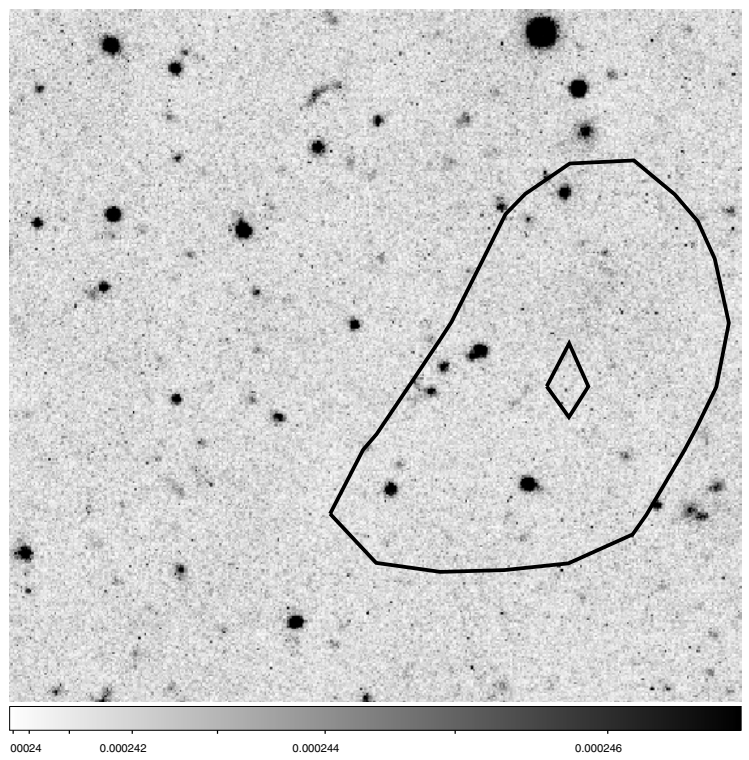

Fig. A.28. $i^{\prime}$ image for $\mathrm{Cl} \mathrm{J} 1216+3318$ observed at ARC (completeness level: $\left.i^{\prime} \sim 23.5\right)$. The field is $1.8 \times 1.8 \operatorname{arcmin}^{2}$. 
C. Adami et al.: An extended SHARC survey, Online Material p 9

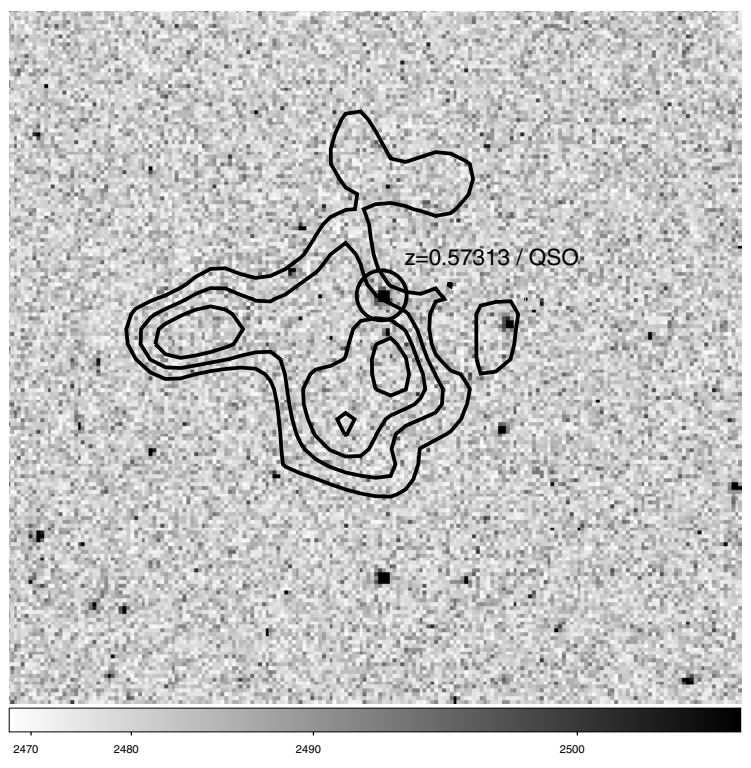

Fig. A.29. $\chi^{2}$ image for $\mathrm{Cl} \mathrm{J} 1234+3755$ built from the SDSS $u, g^{\prime}, r^{\prime}, i^{\prime}$ and $z^{\prime}$ images. The field is $3.7 \times 3.7 \operatorname{arcmin}^{2}$.

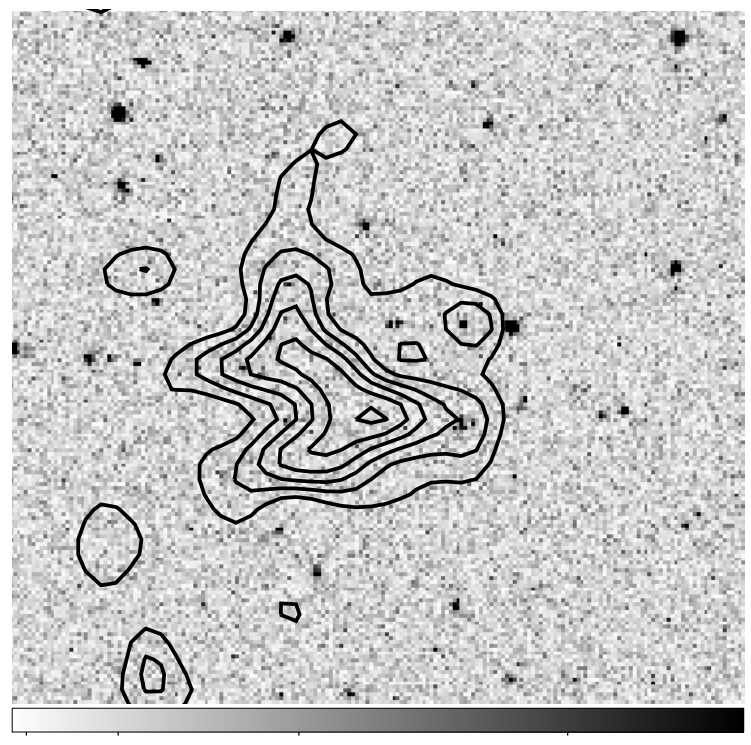

Fig. A.30. $\chi^{2}$ image for Cl J1237+2800 built from the SDSS $u, g^{\prime}, r^{\prime}, i^{\prime}$ and $z^{\prime}$ images. The field is $3.7 \times 3.7 \mathrm{arcmin}^{2}$.

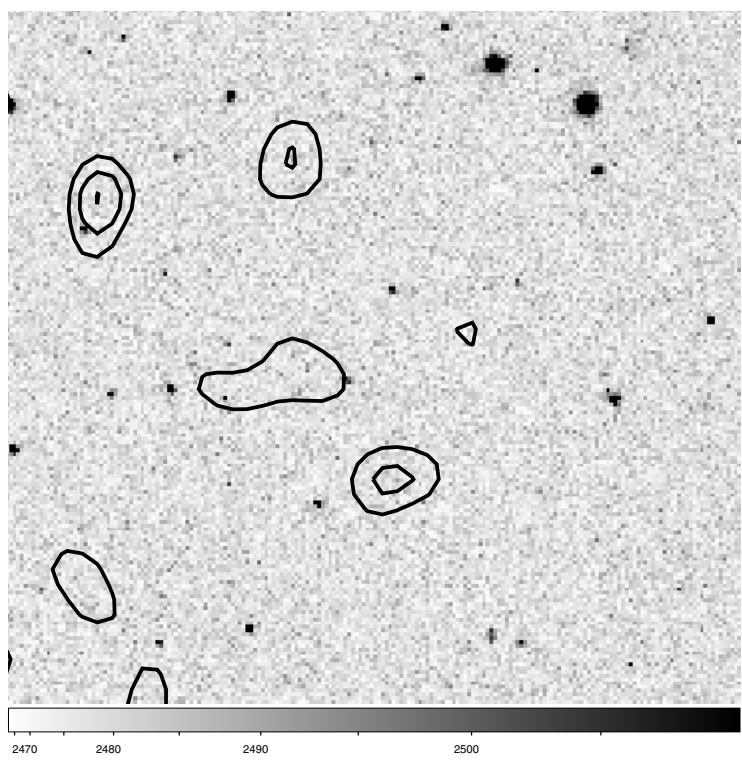

Fig. A.31. $\chi^{2}$ image for $\mathrm{Cl} \mathrm{J} 1259+2547$ built from the SDSS $u, g^{\prime}, r^{\prime}, i^{\prime}$ and $z^{\prime}$ images. The field is $3.7 \times 3.7 \operatorname{arcmin}^{2}$.

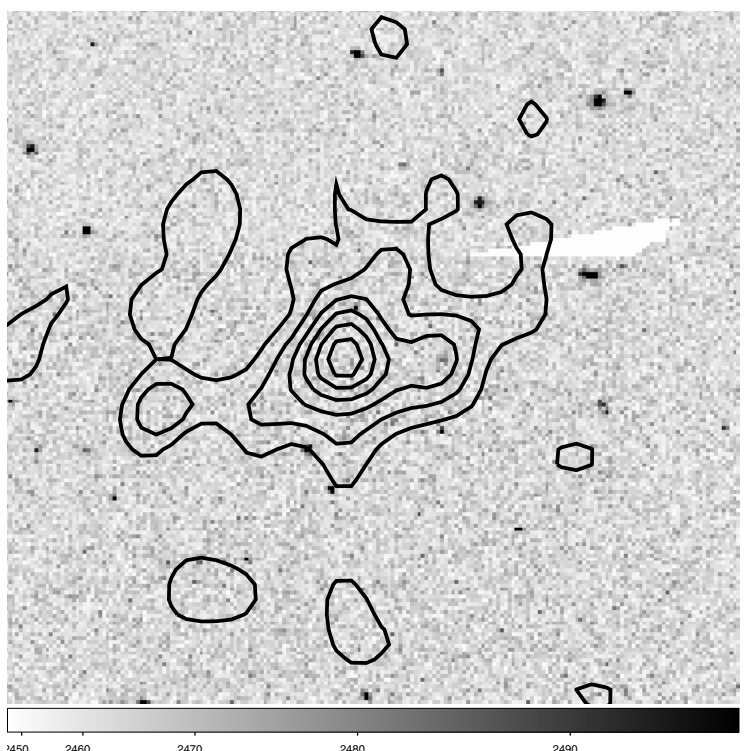

Fig. A.32. $\chi^{2}$ image for Cl J1343+2716 built from the SDSS $u, g^{\prime}, r^{\prime}, i^{\prime}$ and $z^{\prime}$ images. The field is $3.7 \times 3.7 \operatorname{arcmin}^{2}$. 


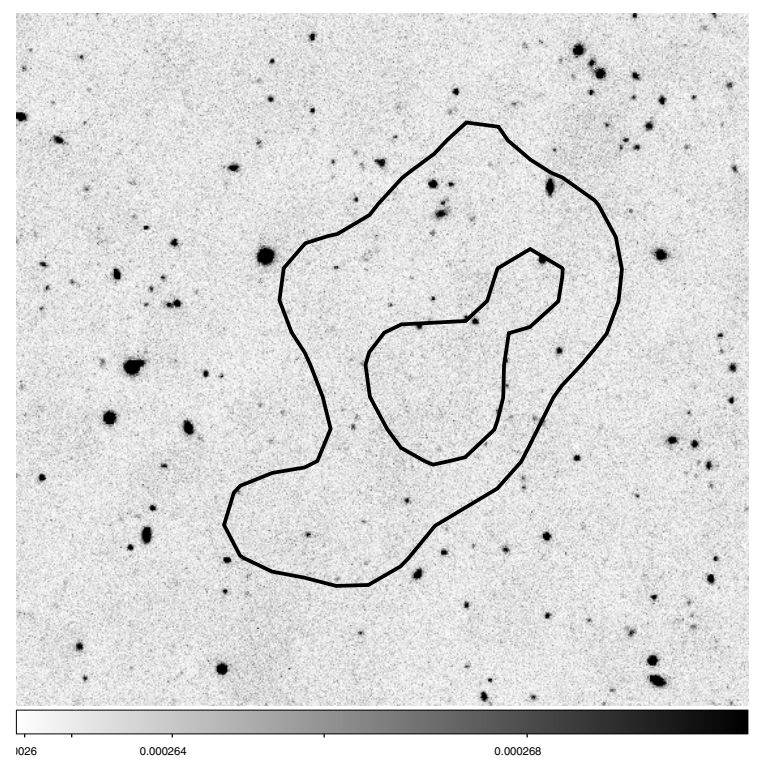

Fig. A.33. $i^{\prime}$ image for $\mathrm{Cl} \mathrm{J} 1350+6028$ observed at ARC (completeness level: $\left.i^{\prime} \sim 23\right)$. The field is $3 \times 3 \operatorname{arcmin}^{2}$.

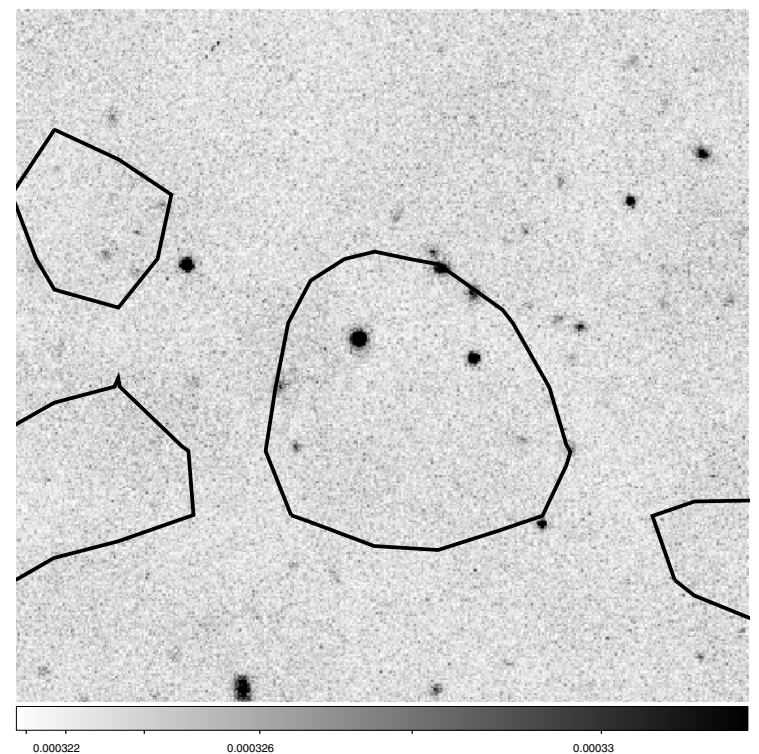

Fig. A.34. $i^{\prime}$ image for $\mathrm{Cl} \mathrm{J} 1411+5933$ observed at ARC (completeness level: $\left.i^{\prime} \sim 23.5\right)$. The field is $1.8 \times 1.8 \mathrm{arcmin}^{2}$.

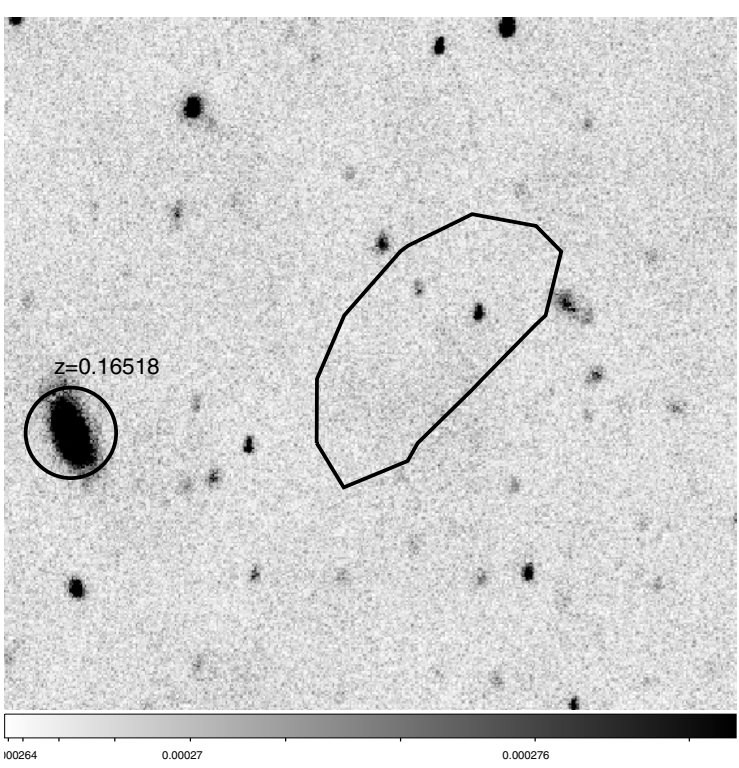

Fig. A.35. $i^{\prime}$ image for $\mathrm{Cl} \mathrm{J} 1514+4351$ observed at ARC (completeness level: $i^{\prime} \sim 22.5$ ). The field is $1.8 \times 1.8 \mathrm{arcmin}^{2}$.

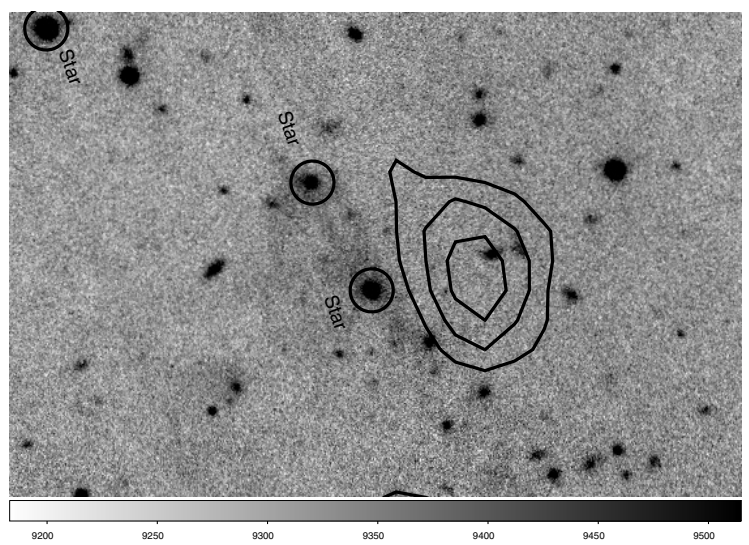

Fig. A.36. $r^{\prime}$ image for $\mathrm{Cl} \mathrm{J} 1651+6107$ observed at Gemini (completeness level: $r^{\prime} \sim 24.5$ ). The field is $1.7 \times 1.1 \mathrm{arcmin}^{2}$. Overlayed X-ray contours are XMM data. Circled objects are stars confirmed by spectroscopy or image analysis. 